\title{
Dust embedded sources at the Galactic Center
}

\section{2 to $4 \mu \mathrm{m}$ imaging and spectroscopy in the central parsec ${ }^{\star}$}

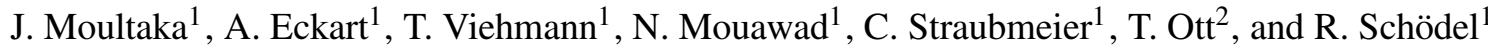 \\ 1 I Physikalishes Institut, Zülpicher Str. 77, 50937 Köln, Germany \\ e-mail: moultaka@ph1.uni-koeln.de \\ 2 Max Planck Institut für extraterrestrische Physik, Giessenbachstrasse, 85748 Garching, Germany
}

Received 4 December 2003 / Accepted 3 June 2004

\begin{abstract}
We present the first $L$-band spectroscopic observations of a dozen stellar sources in the central 0.5 pc of the GC stellar cluster that are bright in the $2-4 \mu \mathrm{m}$ wavelength domain. The $L$-band data were taken with ISAAC at the VLT UT1 (Antu). With the aid of additional $K$-band spectroscopic data we derive the optical depth spectra of the sources after fitting their continuum emission with a single reddened blackbody continuum. We also derive intrinsic source spectra by correcting the line of sight extinction via the optical depth spectrum of a late type star that is most likely not affected by local dust emission or extinction at the Galactic Center. The good agreement between the two approaches shows that the overall variation of the line-of-sight extinction across the central $0.5 \mathrm{pc}$ is $\Delta A_{K} \leq 0.5 \mathrm{mag}$. The extinction-corrected spectra of the hot He-stars resemble pure Rayleigh-Jeans continuum spectra. The intrinsic spectra of all other sources are in agreement with being the result of the continuum emission and absorption features due to the dust in which they are embedded. We interprete both facts as evidence that a significant amount of the absorption takes place within the central parsec of the Galactic Center and is most likely associated with the individual sources there. We find absorption features at $3.0 \mu \mathrm{m}, 3.4 \mu \mathrm{m}$, and $3.48 \mu \mathrm{m}$ wavelength. Correlations between all three features show that they are very likely to arise in the ISM of the central $0.5 \mathrm{pc}$. Spatially highly variable hydrogen emission lines seen towards the individual sources give evidence of the complex density and temperature structure of the mini-spiral. The featureless $K$-band spectra of sources like IRS 21 and IRS $1 \mathrm{~W}$ are consistent with these sources being massive hot stars embedded in the bow shock created by their motion through the dust and gas of the mini-spiral. The bow shock scenario may be applicable to most of the dust-embedded sources in the central stellar cluster. Spectroscopy of high MIR-excess sources $0.5^{\prime \prime}$ north of the IRS 13 complex is largely consistent with them being YSOs. However, a bow-shock nature of these sources cannot be excluded. The $L$-band spectrum at the location of SgrA* closely resembles that of a hot O-type star, such as $\mathrm{S} 2$, which was very close to Sgr $\mathrm{A}^{*}$ at the time of our observations.
\end{abstract}

Key words. Galaxy: center - galaxies: nuclei - infrared: stars - infrared: ISM

\section{Introduction}

Near-infrared diffraction limited imaging over the past 10 years (Eckart \& Genzel 1996; Genzel et al. 1997; Ghez et al. 1998, 2000; Eckart et al. 2002; Schödel et al. 2002, 2003; Ghez et al. 2003) has yielded convincing evidence for a $3-4 \times 10^{6} M_{\odot}$ black hole at the center of the Milky Way. This finding is supported by the discovery of a variable X-ray and NIR source at the position of SgrA* (Baganoff et al. 2001; Genzel et al. 2003a). Most intriguingly, near-infrared imaging and spectroscopic observations have provided evidence for recent star formation in the central parsec of the Milky Way, an environment previously thought hostile to star formation because of the tidal field of the black hole, intense stellar winds, and strong magnetic fields.

* Tables 4-6 and Figs. 2-4, 6, 7, 9-11 are only available in electronic form at http://www. edpsciences.org
At a distance of $8 \mathrm{kpc}$ (Eisenhauer et al. 2003), the Galactic Center is surrounded by a circumnuclear ring of dense gas and dust showing clumpy extinction (Güsten et al. 1987). Inside this ring, there is a central cavity of about 3 pc diameter that contains mainly ionized or atomic gas. The visual extinction estimates towards prominent sources within the central stellar cluster range between 20 and 50 mag with a median around 30 mag (see Rieke et al. 1989; Chan et al. 1997; Scoville et al. 2003). In addition Scoville et al. (2003) showed that the extinction is smoothly distributed across the central 10 to 20 arcsec with no indication of concentrations of extinction on scales of about $1^{\prime \prime}$ to $2^{\prime \prime}$.

The visual extinction by $\sim 30$ mag along the line of sight toward the Galactic Center (GC) is mostly due to the diffuse interstellar medium (ISM) (Lebofsky 1979) and in part to dense molecular gas (Gerakines et al. 1999; de Graauw et al. 1996; Lutz et al. 1996). The absorbing gas is cold (10 K) and 
the abundances of important molecular species are similar to those in the solar neighborhood (Moneti et al. 2001a; Chiar et al. 2000). In addition Blum et al. (1996) and Clénet et al. (2001) concluded that the colours of individual dusty sources within the central stellar cluster contain a substantial contribution from intrinsic reddening.

The entire central parsec of our Galaxy is powered by a cluster of young and massive stars (Blum et al. 1988; Krabbe et al. 1995; Genzel et al. 1996; Eckart et al. 1999; Clénet et al. 2001). Within that cluster the 7 most luminous $\left(L>10^{5.75} L_{\odot}\right)$, moderately hot $\left(T<10^{4.5} \mathrm{~K}\right)$ blue supergiants contribute half of the ionizing luminosity of that region (Najarro et al. 1997; Krabbe et al. 1995; Blum et al. 1995). Such massive and hot stars were also found in dense clusters within the Galactic bulge, i.e. the Arches cluster (Cotera et al. 1992, see also Figer et al. 2002 and references therein) and the Quintuplet cluster (e.g. Figer et al. 1997).

In addition to the massive blue supergiants, a population of dusty sources associated with bright dust emission can be found in the Galactic Center stellar cluster. After initial work by Becklin \& Neugebauer $(1968,1969)$ the first individual mid-infrared sources in the central stellar cluster (among them IRS 1, 3 and others) were reported by Rieke \& Low (1973) and Becklin \& Neugebauer (1975). Later, IRS 1 was resolved into multiple components by Storey \& Allen (1983), Rieke et al. (1989), Simon et al. (1990), and Herbst et al. (1993). Further high resolution imaging by Tollestrup et al. (1989) resolved IRS 6 and IRS 12 into multiple components.

In this paper, we discuss MIR sources that are located well within the central stellar cluster at projected distances from $\mathrm{Sgr} \mathrm{A}^{*}$ of less than $0.5 \mathrm{pc}$ (Fig. 1). Several sources like IRS 1, 3, and 21 are dominated by dust emission and are strong at a wavelength of $10 \mu \mathrm{m}$, whereas the supergiant IRS 7 is brightest at $2.2 \mu \mathrm{m}$. The nature of the dust-enshrouded sources is still unknown. Among the best studied cases is IRS 21 , which is strongly polarized ( $17 \%$ at $2 \mu \mathrm{m}$; Eckart et al. 1995; Ott et al. 1999; Krabbe et al. 1995). Initially, Gezari et al. (1985) suggested that IRS 21 is an externally heated, highdensity dust clump. Given the MIR excess and the featureless NIR spectra several other classifications have been proposed, including an embedded early-type star and a protostar (Blum et al. 1988; Krabbe et al. 1995; Genzel et al. 1996; Clénet et al. 2001). Tanner et al. (2002) suggest that IRS 21 is an optically thick dust shell surrounding a mass-losing source, such as a dusty recently formed WC9 Wolf-Rayet star. Tanner et al. (2002, 2003) indicate that the extended dust emission of most of the central sources is consistent with bow shocks created by the motion of massive hot stars through the dust and gas of the mini-spiral.

One way of investigating the nature of these bright NIR/MIR sources is by imaging and spectroscopy in the 2 to $4 \mu \mathrm{m}$ wavelength range. In addition to hydrogen and helium recombination lines, this wavelength domain is dominated by strong absorption features due to abundant molecules $\left(\mathrm{NH}_{3}\right.$, $\mathrm{CH}_{3} \mathrm{OH}, \mathrm{H}_{2} \mathrm{O}, \mathrm{CO}, \mathrm{CO}_{2}$ etc.), functional groups (like $\mathrm{NH}_{2}$, $\mathrm{CH}_{2}$ ), and ices. Here $\mathrm{H}_{2} \mathrm{O}$ ice enriched with molecular material is of special importance. Liquid, crystalline, amorphous water ice as well as trapped water ice in $\mathrm{SiO}$ condensate

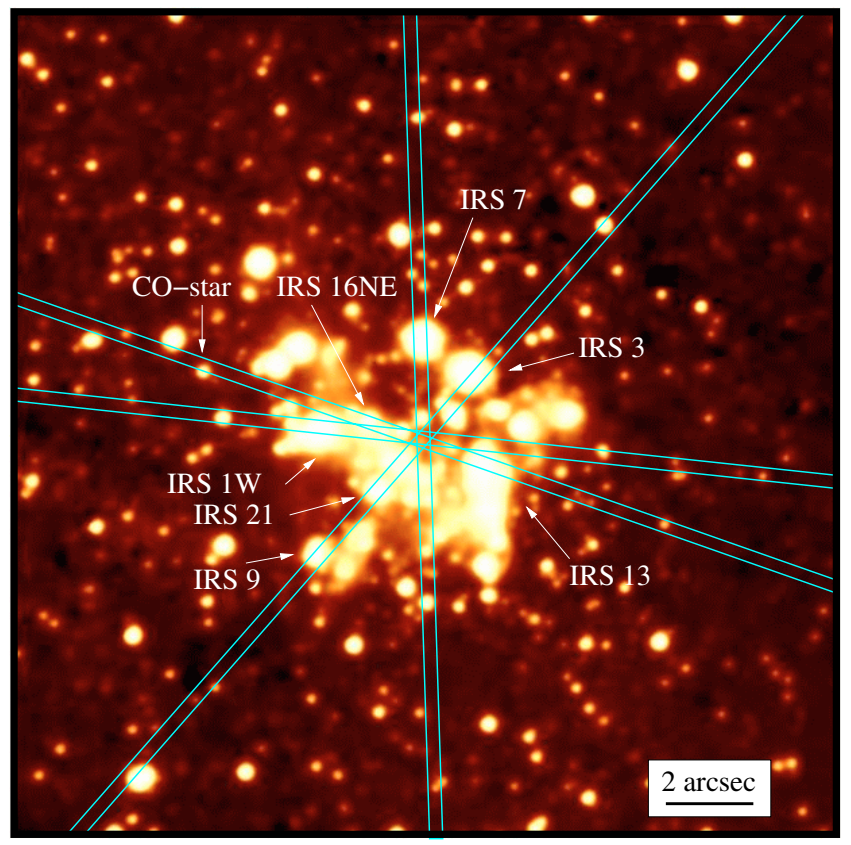

Fig. 1. L-band image as obtained with ISAAC on the VLT UT1. The four slit positions chosen for the spectroscopic observations are also shown. They all include the position of SgrA*. The position of the CO-star used for the calibration along the line of sight is shown as well.

(Wada et al. 1991) give rise to a rich variety in shapes of a prominent feature with its deepest absorption at 2.94-3.00 $\mu \mathrm{m}$ (e.g. Wada et al. 1991). The variety in shapes of the water ice feature is dependent not only on temperature, but also on annealing history and on the ice composition etc. (Hagen et al. 1983; Tielens \& Hagen 1982; Tielens et al. 1983; Kitta \& Kratschmer 1983; Hudgins et al. 1993; Maldoni et al. 1998).

The emission of dust and the absorption features of ices are important diagnostic tools for the investigation of the interstellar medium and circumstellar environments of individual sources.

Infrared sources towards the Galactic Center show a wealth of ice absorption features (Butchart et al. 1986; Sandford et al. 1991) indicative of a broad range of organic material mostly in the diffuse interstellar medium. Aliphatic hydrocarbons are characterized by their $\mathrm{CH}_{2}$ (methylene) and $\mathrm{CH}_{3}$ (methyl) stretching modes around $3.4 \mu \mathrm{m}$ (Sandford et al. 1991; Pendleton et al. 1994). Aromatic hydrocarbons are detected via their $\mathrm{CH}$ and $\mathrm{CC}$ stretching modes at 3.28 and $6.2 \mu \mathrm{m}$. (Chiar et al. 2000; Pendleton et al. 1994). An absorption feature at $3.25 \mu \mathrm{m}$ has been found towards dense molecular clouds. It is attributed to aromatic hydrocarbon molecules at low temperatures (Sellgren et al. 1995; Brooke et al. 1999). Differences in the exact central wavelength and profile width of the absorption near $3.3 \mu \mathrm{m}$ are mostly attributed to differences in temperature and/or carrier of the absorbing molecules in these regions.

In this paper we present 3 to $4 \mu \mathrm{m}$ imaging and spectroscopy data combined with near-infrared $2.2 \mu \mathrm{m}$ spectroscopy of the strongest mid-infrared sources in the central stellar cluster. In addition to the previously published $L$-band observations of IRS $1 \mathrm{~W}$, IRS 3 and IRS 7 we provide the 
first $L$-band spectra of 9 other MIR sources: IRS 9, IRS 13, IRS 13N, IRS 21, IRS 29 and IRS $16 \mathrm{C}, \mathrm{CC}, \mathrm{NE}$ and SW. These data on sources located in the central $0.5 \mathrm{pc}$ of the GC enable us to study the properties of the local interstellar medium and of circumstellar matter in this region.

\section{Observations and data reduction}

To investigate the nature of the dust-embedded sources within the central $0.5 \mathrm{pc}$ of the nuclear stellar cluster we used nearand mid-infrared imaging and spectroscopy. In the following we describe the instrumentation and the data reduction.

\subsection{MIR observations and data reduction}

Mid-infrared imaging and spectroscopy was obtained using the ISAAC instrument on the ESO Very Large Telescope (VLT) unit telescope UT1 (Antu), at the Paranal observatory in Chile. We have performed spectroscopic and imaging observations of the Galactic Center during May 23-30, 2002, as part of a monitoring program of SgrA* (Eckart et al. 2003; Baganoff et al. 2003). All the data reduction has been performed using routines from the IRAF and MIDAS software packages.

For the imaging in the $L$-band, paired flat fielded images at different chopper throws $\left(18^{\prime \prime} \pm 2^{\prime \prime}\right)$ and chopping position angles $\left(0^{\circ}\right.$ to $\left.180^{\circ}\right)$ were subtracted from each other, resulting in frames containing a positive and a (shifted) negative image. The frames were then shifted to a common reference point that coincides with a positive image of a source. Subsequently, frames belonging to the same batch, i.e. taken sequentially with identical or different chopper throws and/or chopping angles were combined by calculating the median. Since the images were moved to a common reference point this procedure eliminates the negative "shadows" generated by the subtractions. This procedure also effectively removes cosmic rays and bad pixel structures. Such a batch typically consists of up to $40 \mathrm{im}$ ages, and each resulting combined image covers an integration time of approximately $35 \mathrm{~min}$. For the present investigation we used the images with the best seeing and image quality with an angular resolution of about $0.4^{\prime \prime}$. The absolute $L$-band flux calibration (see magnitudes listed in Table 1) was performed using the fluxes of several bright well isolated objects also measured by Clénet et al. (2001).

The spectroscopic observations were performed with the long-wavelength (LWS3) and low resolution (LW) mode using the SL filter covering the wavelength range of $2.7 \mu \mathrm{m}-4.2 \mu \mathrm{m}$ and $4.4 \mu \mathrm{m}-5.1 \mu \mathrm{m}$, respectively. The use of a $0.6^{\prime \prime}$ slit width implied a spectral resolution $(R=\lambda / \Delta \lambda)$ of $R=600$ in that wavelength domain. The seeing at this time was in the range between 0.4 and 0.9 arcsec. To compensate for the thermal background, separate chopped observations were carried out using chopper throws of $\sim 18$ arcsec and random nodding within 2 arcsec along the slit. We adopted 4 different slit positions that all included the SgrA* location (see Fig. 1). The resulting images were divided by flat-fields, corrected for cosmic rays, for sky lines and for dispersion-related distortion. The wavelength calibration was performed using a xenon-argon lamp.
Table 1. Results from fitting a single reddened blackbody to the $K$ and $L$-band spectra. In addition to the blackbody temperature and the $K$-band extinction obtained from the fitting procedure, we list the $L$-band magnitudes $( \pm 0.2$ mag uncertainty) we derived from our images.

\begin{tabular}{rccc}
\hline \hline Source & $\begin{array}{c}\text { Blackbody } \\
\text { temperature }\end{array}$ & $A_{K}$ & $\begin{array}{c}L \text {-band } \\
\text { magnitude }\end{array}$ \\
\hline IRS 1W & 900 & 3.30 & 5.8 \\
IRS 3 & 800 & 4.20 & 5.3 \\
IRS 7 & 2100 & 3.55 & 5.0 \\
IRS 9 & 1900 & 3.10 & 7.4 \\
IRS 21 & 1200 & 3.95 & 6.8 \\
IRS 29 & 1600 & 3.70 & 7.4 \\
IRS 16C & 22600 & 3.45 & 8.6 \\
IRS 16CC & 20200 & 4.00 & 9.0 \\
IRS 16NE & 22400 & 3.25 & 7.7 \\
IRS 16SW & 22500 & 3.25 & 8.4 \\
IRS 13 & 1300 & 3.00 & 6.7 \\
IRS 13N & 1000 & 3.90 & 9.76 \\
\hline
\end{tabular}

Two chopped frames (with shifted image positions) were then subtracted from each other to provide a single frame containing two negative trace images and a positive one with twice the intensity of the negative images. After extraction of the individual source spectra they were corrected for wavelength dependent sensitivity, atmospheric transmission, and telluric lines using two standard stars HD 194636 (B4V) and HD 148703 (B2III-IV).

The spectra were normalized to the given $K$ - and $L$-band magnitudes listed in Table 1.

\subsection{NIR Imaging spectroscopy and data reduction}

The near-infrared data in the $K$-band $(2.2 \mu \mathrm{m})$ were obtained via integral field spectroscopy using the imaging spectrograph 3D (Weitzel et al. 1996) combined with the tip/tilt corrector ROGUE (Thatte et al. 1995) at the ESO/MPG 2.2 m located at La Silla. This instrument allows observations of a continuous 2-dimensional field $(16 \times 16$ pixels $)$ while providing spectral information for each spatial image element. These seeing-limited observations were done with a pixel scale of $0.3^{\prime \prime}$ resulting in a field of view of $4.8^{\prime \prime} \times 4.8^{\prime \prime}$ for individual pointings. Using a spectral resolution of $R=2000$ each half of the $K$-band had to be covered separately.

The observations were centered on the IRS 16 cluster. In March 1996, the central parsec was observed with a spectral coverage from 1.97 to $2.21 \mu \mathrm{m}$ (lower half of the $K$-band). In total 17 different but overlapping regions were observed. The total sky area covered amounts to $16^{\prime \prime}$ in the East-West and 10" in the North-South direction. The upper half of the $K$-band from 2.18 to $2.45 \mu \mathrm{m}$ was observed during a second observing run in April 1996. Here a total of 52 overlapping regions was observed. This resulted in an area of $25^{\prime \prime}$ in the 
North-South direction and 20" in East-West. For further details of the observations see Genzel et al. (1997), Ott et al. (2003).

To calibrate the wavelength scale, spectral lamp data (argon lamps in this case) were taken at the beginning or the end of each observing night. As a further step, calibration sources with a known spectrum were observed at a similar airmass as the Galactic Center. These standard stars were divided by a spectrum of the same stellar type (Kleinmann \& Hall 1986) to remove stellar features, resulting in an atmospheric transmission spectrum. The source data were then divided by this spectrum.

\section{Extinction correction}

The patchy extinction towards the GC stellar cluster (see e.g. Scoville et al. 2003) demands a careful calibration of spectral data. Therefore, the correction for the extinction along the line of sight has been carried out using the method described in Sect. 3.1 and tested using another, independent method described in Sect. 3.2. A cross-check shows that both our two approaches agree very well (see the results in Sect. 3.2.2 and the conclusion (Sect. 3.3) at the end of this section) and that the extraction of the optical depth spectra described in Sect. 4 can be done safely.

\subsection{Fitting blackbody emission to the 2 to $4 \mu \mathrm{m}$ spectral continua}

\subsubsection{Method}

To estimate the extinction towards the individual sources and to determine the approximate continuum shape of the observed spectra, we have performed simultaneous fits of our $K$ and $L$-band data with single, reddened blackbody spectra. In this process we have considered the continuum emission, fitted locally around $2.2 \mu \mathrm{m}, 3.75 \mu \mathrm{m}$, and $4.17 \mu \mathrm{m}$ wavelength, as representing the intrinsic, reddened continuum of the sources. To find the best fit, the temperature of the blackbody was allowed to vary within given limits with a step size of $100 \mathrm{~K}$. For the helium stars these limits were 20000 and $26000 \mathrm{~K}$ (Najarro et al. 1997). For the remaining objects the limits were $200 \mathrm{~K}$ and $4000 \mathrm{~K}$. We allowed the $K$-band absorption $A_{K}$ to vary over a range between 2.7 and $4.5 \mathrm{mag}$ with a step sizes of 0.05 mag assuming the extinction law $A_{\lambda} \sim \lambda^{-1.8}$ stated by Martin \& Whittet (1990). We extended the fitting range towards higher $A_{K}$ values to allow a search for higher extinction towards the dusty sources. In general the fitting is not very sensitive with respect to the determination of $A_{K}$ due to the strong $\mathrm{H}_{2} \mathrm{O}$ absorption feature that dominates almost the entire $L$-band.

There are several potential problems that one has to consider when using this procedure (see also discussion in Chiar et al. 2002):

We have to take into account that our $K$ - and $L$-band data have not been taken at the same epochs and in some cases their relative calibration may be affected by variability. Some sources in the central parsec of the Milky Way are variable on time scales of months to years (Ott et al. 1999;
Blum et al. 1996; Tamura et al. 1994, 1996). To achieve a successful combined fit over both bands we therefore used different overall flux calibrations of the spectra. For the $K$-band fluxes, we considered data from 5 different references: Becklin et al. (1978), Tollestrup et al. (1989), Blum et al. (1996), Ott et al. (1999), and Clénet et al. (2001). The observations of Clénet et al. (2001) are the most recent of the four references and have been obtained with Adaptive Optics. For this reason, we have preferentially used the values given in Clénet et al. (2001) for the fitting procedure. However, for some sources a better fit was obtained when a different flux calibration was used. This was the case for IRS 7, IRS 9, the stars in the IRS 16 complex as well as IRS 13. IRS 7 and IRS 9 are variable as shown in Ott et al. (1999), Blum et al. (1996), and Tamura et al. (1994, 1996). The relative flux density calibration from Becklin et al. (1978) results in the best fits for our data, since their observations in the $K$ - and $L$-bands were performed in the same year. The IRS 13 complex consists of at least three bright members, E1, E2, and E3 (Paumard et al. 2001; Maillard et al. 2003). They contain the hottest and most luminous star in the entire region (Najarro et al. 1997). The IRS 13 complex therefore is very likely to be variable in flux density. In this case the fitting results using the $K$-band fluxes by Blum et al. (1996) were best.

The blackbody temperatures and $K$-band extinction resulting from the best fits of the spectra are listed in Table 1.

Moreover, most of the spectra are heavily affected by broad absorption features and it is difficult to determine a clear measure of the underlying continuum emission. Also, the assumption of a single temperature blackbody continuum can only be taken as a first approximation. On the other hand, reddened multi-temperature models quickly result in a larger number of not well determined parameters (e.g. temperature and relative flux density contribution for each component).

The resulting best fits of the individual spectra with a single blackbody are shown in Figs. 2 to 4.

\subsubsection{Results}

We list the resulting parameters of the fits in Table 1 and the spectra with the corresponding blackbody curves in Figs. 2-4. The temperatures and the $K$-band extinctions derived by the fitting procedure agree well with those found by other authors: The He stars of the IRS 16 complex are best fitted with temperatures that are similar to those found by Najarro et al. (1997). IRS 7 and IRS 9 have typical temperatures of late-type supergiants or giant stars (Chiar et al. 2002; Ott et al. 1999) and the remaining dusty sources are well fitted by blackbody continua of typical temperatures for hot dust (Tanner et al. 2002; Gezari et al. 1996; Genzel et al. 1997; Blum et al. 1996, and others).

The overall shapes we obtain by fitting single-temperature blackbody curves are reasonable and compare favorably to those of Chiar et al. (2002) (especially in the case of the three sources in common, IRS 1W, IRS 3 and IRS 7). In addition, they are supported by an independent procedure to calibrate the line of sight absorption described in the following section (Sect. 3.2). Also, the inclusion of the $K$-band spectra, which 
were missing in the work by Chiar et al. (2002) makes it possible to better judge the quality of the fitting procedure by comparison with the $K$-band continuum fluxes and spectral shapes, especially for the three sources in common (IRS $1 \mathrm{~W}$, IRS 3 and IRS 7).

Despite the improvements in the spectral fitting, there are some mismatches we want to comment on:

The fit of the IRS 1W spectrum shown in Fig. 2 does not match the $K$-band spectrum of this source and shows a continuum level mismatch in the $3.8 \mu \mathrm{m}$ to $4.0 \mu \mathrm{m}$ region that has no physical significance. This is certainly due to a non-consistency between the flux calibrations in the $K$ - and $L$-spectral bands. For this reason, we decided to set an upper and a lower limit to the fit by performing a fit that matched the $K$-band spectrum perfectly without caring about the adjustment at the red part of the spectrum on the one hand, and another fit compensating the non-physical mismatch at $3.8 \mu \mathrm{m}$.

An absorption at $\sim 4.2 \mu \mathrm{m}$ appears in the spectra of IRS 9 and IRS 29. This depression in flux is hardly an artefact of the data reduction, since we used the same procedures as for the other sources. We have no scientific explanation for that depression but considered it as real. Also, both in shape and center wavelength, it does not seem to be consistent with the closest (at $4.27 \mu \mathrm{m}$ ) strong absorption line due to the stretching mode resonance of solid $\mathrm{CO}_{2}$ also observed towards the dust shells of some Young Stellar Objects (de Graauw et al. 1996). The presence of this depression does not affect the results and conclusions in this paper.

Concerning the IRS 16 objects, none of the fits was very satisfactory towards the red part of the $L$-band spectrum while all of them match well the $K$-band spectra. It is obvious that this is not due to the fitting procedure itself but to the shape of the $L$-band spectra of these sources which are very flat (except for the IRS $16 \mathrm{NE}$ case where the shape matches better the overall shape of a Rayleigh-Jeans spectrum). These spectra have been reduced in the same way as all the other spectra with the same flux calibrator stars and thus the shape of the spectra is real.

The origin of this behavior is not clear. It may be due to the fact that these sources are the least contaminated by local dust emission features and are likely the most susceptible to variations in the wavelength dependent line of sight extinction, and to properties of the associated material. If for instance - compared to the intrinsic, local absorptions of the dusty sources the line of sight absorption is dominated by amorphous $\mathrm{H}_{2} \mathrm{O}$ ice (Wada et al. 1991), the corresponding line center and strength of the red wing would be shifted towards the red. As a result the slope of the reddened continuum simultaneously fitting the $K$ and $L$-band continuum would be systematically too large.

\subsection{Determining the wavelength-dependent line of sight extinction}

\subsubsection{Method}

The calibration procedure described in this section is supported by the finding of Scoville et al. (2003) that the extinction shows a smooth distribution across the central 10 to 20 arcsec with no indication of concentrations of extinction on scales of about $1^{\prime \prime}$ to $2^{\prime \prime}$. Furthermore Blum et al. (1996) and Clénet et al. (2001) concluded that the colours of the sources within the central stellar cluster are due to both intrinsic and foreground reddening.

One of the slit settings we adopted for our observations runs through a late-type star shown in Fig. 1. This star shows clear $2.3 \mu \mathrm{m} \mathrm{CO}$ absorption band-heads in its $K$-band spectrum (see Fig. 6); therefore, we hereafter call it "CO-star" (e.g. Eckart et al. 1995). It is located at a projected distance of 12.6" $(\sim 0.5 \mathrm{pc})$ from the center, is well outside the mini-spiral emission and does not show excess emission at wavelengths of $3 \mu \mathrm{m}$ or longer (see the $L$-band spectrum in Fig. 7).

Therefore we can safely assume that this star is largely free of local reddening and that its spectrum is mostly affected by the line of sight extinction along the $8 \mathrm{kpc}$ towards the Galactic Center.

We assume that the $L$-band spectrum of the CO-star can be represented by a $3600 \mathrm{~K}$ (M0-M3 spectral type) blackbody spectrum. If one matches the $\sim 4.2 \mu$ m flux densities of the measured $L$-band spectrum to the theoretical continuum blackbody spectrum then the ratio between the two spectra provides a measure of the wavelength-dependent extinction in the $L$-band of the spectrum due to the interstellar medium along the line of sight. The corresponding wavelength distribution of the optical depth is shown in Fig. 8. If we correct this spectrum with the continuum optical depth value at $4.2 \mu \mathrm{m}$ of $\tau=1.09$ (derived by interpolation between the expected $L$ - and $M$-band extinctions given by Rieke \& Lebofsky 1985) the mean optical depth would be of the order of 1.64. This result is consistent with the value of 1.55 obtained by Rieke \& Lebofsky (1985) for the $L$-band optical depth. We have divided the spectra of all remaining objects by this extinction spectrum (of which the wavelength distribution is shown in Fig. 8). The resulting spectra then represent probably more closely the source spectra as seen at the location of the Galactic Center - corrected for wavelength-dependent absorption along the line of sight towards the Galactic Center.

\subsubsection{Results}

We show the spectra after correction for wavelength-dependent absorption together with our $L$-band image in Fig. 5.

In Figs. 9 to 11, the spectra corrected for the measured wavelength-dependent line of sight extinction as determined from the CO-star are shown together with the blackbody continuum spectra that were derived by fitting the non-corrected spectra with a single reddened blackbody (Sect. 3.1).

We find that they can be grouped into three classes represented by the three figures.

- I. As shown in Fig. 11, the spectral shapes of the hot stars found in the IRS16 complex are fitted well by RayleighJeans continuum spectra i.e. high temperature blackbody spectra (listed in Table 1).

The slight mismatch between the blackbody spectra and the calibrated observed spectra in the red part of the $L$-band wavelength range corresponds to the one between the uncorrected spectra and the reddened blackbody continua 


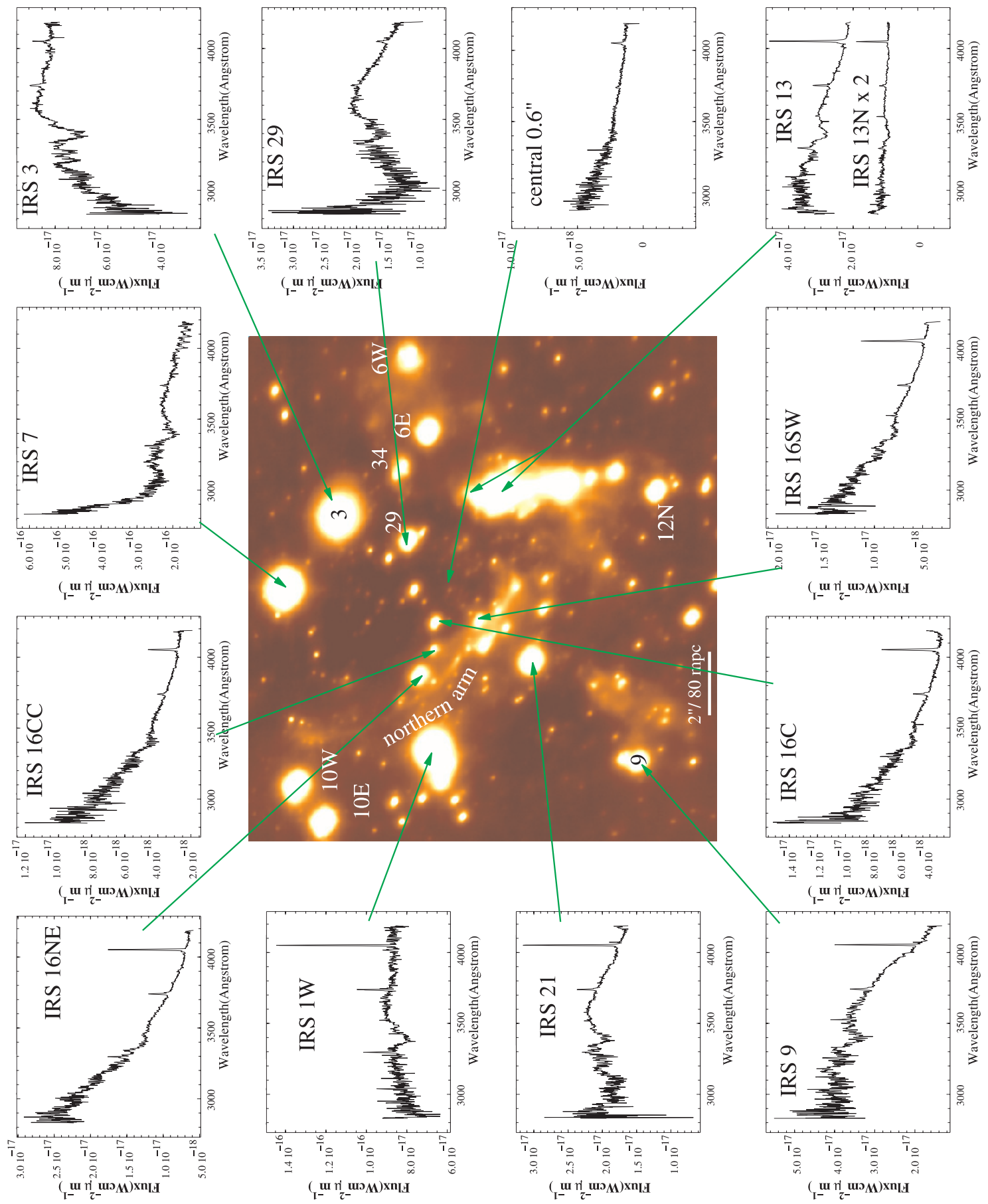

Fig. 5. Our ISAAC $L$-band map combined with the spectra of the central sources corrected for the line of sight extinction.

shown in Fig. 4. Its origin is, thus, probably the same as mentioned in the previous section.

- II. The corrected spectra of IRS 1W, IRS 3, IRS 13N and IRS 21 (Fig. 9) are flat in the red part with indications of continuum absorption at wavelengths shortward of $3.2 \mu \mathrm{m}$. The spectra can be roughly explained by a $T \leq 1500 \mathrm{~K}$ blackbody continuum, with deviations at wavelengths $\leq 3.5 \mu \mathrm{m}$ due to the red wing of the $3.0 \mu \mathrm{m}$ ice absorption feature.

- III. Finally, in addition to strong $\mathrm{H}_{2} \mathrm{O}$ ice absorption the spectra of IRS 7, IRS 9, IRS 13, and IRS 29 (Fig. 10), show longward of $3.2 \mu \mathrm{m}$ the characteristic shape of a $T \sim$ $1500 \mathrm{~K}$ to $2000 \mathrm{~K}$ continuum. The absorption at $4.2 \mu \mathrm{m}$ corresponds to that distinguished in the observed spectrum of Fig. 3.

\subsection{Conclusion on the extinction correction}

The comparison of the extinction-corrected spectra (using the $\mathrm{CO}$-star) and the blackbody continuum spectra with temperatures equal to the ones obtained by the fitting procedure (Sect. 3.1 and Table 1) shows that the overall shape of the 


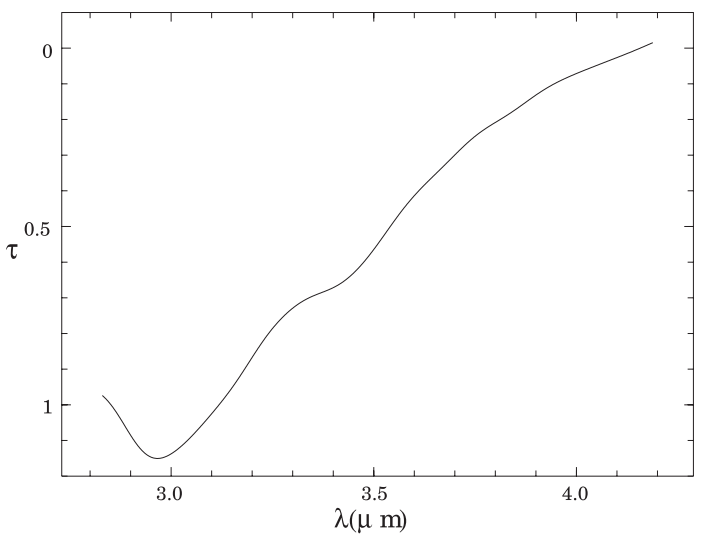

Fig. 8. Wavelength distribution of the line of sight extinction towards the Galactic Center.

corrected spectra is consistent with the blackbody curve for the corresponding temperature. The dusty sources are fitted well with temperatures of the order of $\sim 1000 \mathrm{~K}$ and the global spectral shapes of the hot Helium stars in the IRS16 complex closely resemble pure Rayleigh-Jeans spectra. This implies that the $L$-band wavelength distribution of the optical depth of Fig. 8 is consistent with the extinction law of Martin $\&$ Whittet (1990) (see Sect. 3.1) and with the known absorption value $2.7 \leq A_{K} \leq 4$ toward the Galactic Center (Rieke $\&$ Lebofsky 1985). Consequently, the fitting procedure carried out in Sect. 3.1 is reliable and one can use the reddened continua obtained in that section to derive optical depth spectra. The extraction of the optical depth spectra is described in the next section. In the following, we do not make use of the corrected spectra obtained in Sect. 3.2 because small absorption features could still be present in the spectrum of the correction CO-star which may affect the optical depth measurements.

In addition, the good agreement between the two calibration procedures implies that the overall variation in extinction across the central $0.5 \mathrm{pc}$ of the Milky Way cannot be much larger than $\Delta A_{K}= \pm 0.5 \mathrm{mag}$. This is consistent with the results by Scoville et al. (2003) who derived extinction estimates from the $\mathrm{P} \alpha / 6 \mathrm{~cm}$ radio continuum and the $\mathrm{P} \alpha / \mathrm{H} 92 \alpha$ line emission over this central region. These estimates result in a distribution which is smooth on the $\sim 1^{\prime \prime}$ scale. This supports the assumption that the excess extinction seen towards some of the sources must in fact be associated with the individual objects rather than with the diffuse ISM (see Blum et al. 1996; Clénet et al. 2001).

\section{Optical depth spectra}

\subsection{Extraction of optical depth spectra}

As has been pointed out in the previous section, the optical depth spectra of the Galactic Center sources can be obtained using the reddened blackbody continua of the fitting procedure (Sect. 3.1 and Figs. 2 to 4).

With the results of the blackbody fitting (Table 1), we have derived the optical depth spectra of the sources using the equation $F_{\text {obs }}=F_{\text {intr }} \mathrm{e}^{(-\tau)}$ where $\tau$ is the optical depth, and $F_{\text {obs }}$ and $F_{\text {intr }}$ are the observed and intrinsic fluxes, respectively. All

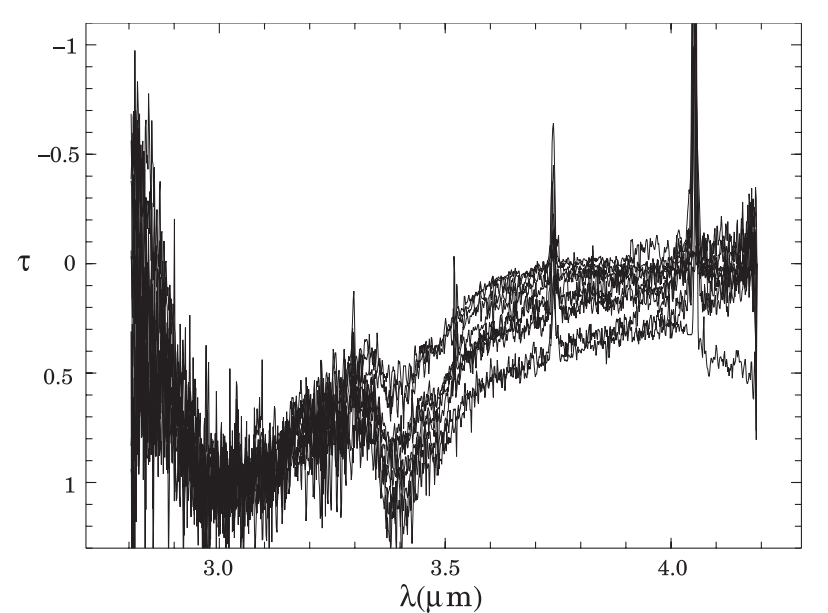

Fig. 12. Optical depths of the Galactic Center sources normalized to unity at $3.0 \mu \mathrm{m}$.

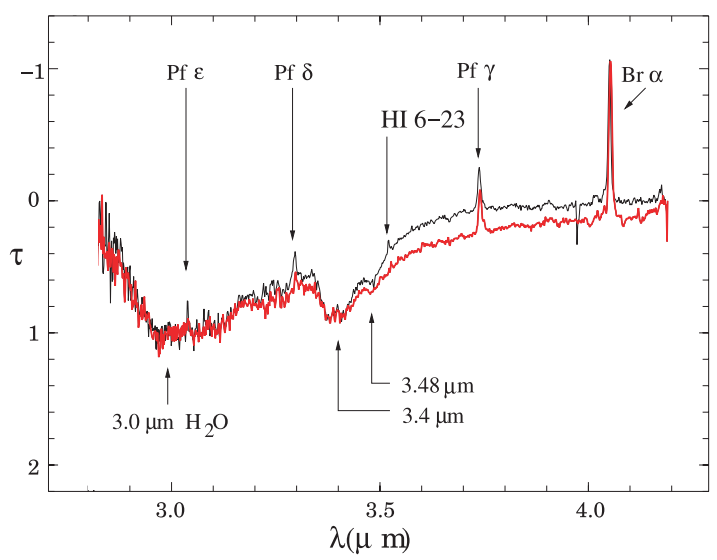

Fig. 13. Mean $L$-band normalized spectra. The black and grey (or red in the colour version) spectra are obtained by averaging the dusty sources and the stellar (He-stars, IRS 7 and IRS 9) optical depth spectra, respectively.

the spectra are shown in Fig. 12 where they have been normalised to unity at $3.0 \mu \mathrm{m}$. Mean optical depth spectra for the dust-enshrouded sources and the non-obscured sources are shown in Fig. 13. This figure shows very clearly three absorption features at $3.0 \mu \mathrm{m}, 3.4 \mu \mathrm{m}$ and $3.48 \mu \mathrm{m}$. From Fig. 12, it is evident that the absorption depths of the $3.4 \mu \mathrm{m}, 3.48 \mu \mathrm{m}$ and $3.0 \mu \mathrm{m}$ features vary from source to source.

Mean spectra of the hydrocarbon feature at $3.4 \mu \mathrm{m}$ and the ice feature at $3.0 \mu \mathrm{m}$ have been constructed in the same iterative way as in Chiar et al. (2002). The optical depth spectrum of IRS 29 shows the smallest $3.4 \mu \mathrm{m}$ absorption in comparison to the $3.0 \mu \mathrm{m}$ feature. A first mean spectrum of the $3.4 \mu \mathrm{m}$ feature was obtained by subtracting the optical depth spectrum of IRS 29 from all other spectra. Then the average of the residual spectra was derived. The mean water profile spectrum is then obtained by subtracting the average spectrum of the $3.4 \mu \mathrm{m}$ hydrocarbon feature multiplied by a free scaling parameter. This parameter is adjusted such that after subtraction of the average spectrum of the hydrocarbon feature from the mean source spectrum a featureless spectrum is obtained in the $3.4 \mu \mathrm{m}$ region. Finally, the spectrum of the $3.4 \mu \mathrm{m}$ 


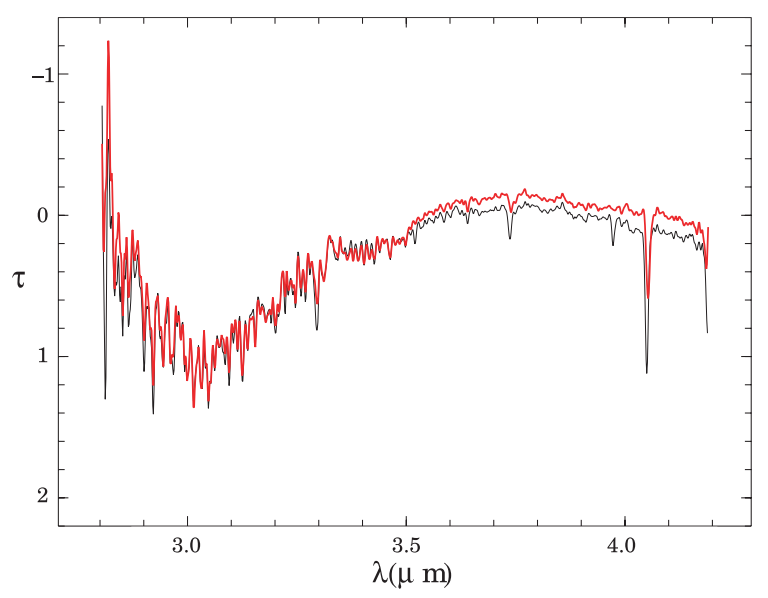

Fig. 14. Mean isolated and normalized ice spectra of the dusty sources (in black) and He-stars, IRS 7 and IRS 9 sources (in grey, or red in the colour version).

hydrocarbon feature was deduced by subtracting the mean $3.0 \mu \mathrm{m}$ spectrum from all the spectra and averaging the residuals. The resulting spectra are shown in Figs. 14 and 15 .

The most reliable results were obtained for the dustenshrouded sources. As a consequence of the successful continuum fits described in Sect. 3.1, the absorption spectra level out at $\tau \sim 0.0$ for wavelengths well above and well below the corresponding absorption features. For the hot He-stars in the IRS16 cluster the situation is different. Here the continuum fits were less successful (see discussion in Sect. 3.1) and the derived profiles of the absorption features are less reliable towards the long wavelength end of the spectra. This is less severe for the $3.0 \mu \mathrm{m}$ feature since in this case only the continuum longward of $\sim 3.5 \mu \mathrm{m}$ is affected.

\subsection{Characteristics of features in the optical depth spectra}

An examination of the optical depth spectra reveals information on the origin and physical conditions of the absorbing material in the central parsec of the GC stellar cluster. We distinguish the following features:

- The $3.0 \mu \mathrm{m}$ feature: the dusty sources (which are located in the minispiral area, like IRS $1 \mathrm{~W}, 3,13,13 \mathrm{~N}$ and 29) and hot He-stars (i.e. showing He emission lines in their $K$-band spectra which is the case for the IRS 16 sources, see Najarro et al. 1997) make it possible to derive both the linewidth and line minimum of the $3.0 \mu \mathrm{m}$ absorption feature. Neither quantity is affected by the slight continuum mismatch at wavelengths above $3.5 \mu \mathrm{m}$ for the He-stars. The $3.0 \mu \mathrm{m}$ absorption feature is associated with interstellar $\mathrm{H}_{2} \mathrm{O}$ ice in molecular clouds (Gillett \& Forrest 1973). Laboratory experiments suggest that the shape of the observed ice absorption (which is deepest at about $2.95 \mu \mathrm{m}$ in IRS 7 for example) toward the Galactic Center corresponds to trapped water ice in $\mathrm{SiO}$ condensates (Wada et al. 1991).

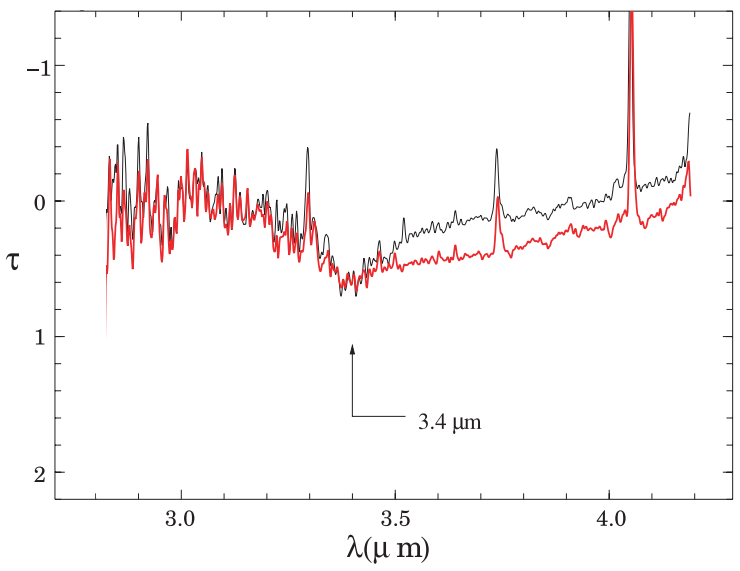

Fig. 15. Mean isolated and normalized $3.4 \mu \mathrm{m}$ feature of the dusty sources (in black) and He-stars, IRS 7 and IRS 9 sources (in grey, or red in the colour version). The location of the $3.3 \mu \mathrm{m}$ feature is indicated.

Our mean absorption spectrum (Fig. 14) shows, however, that in our observations the deepest absorption occurs $\sim 0.05 \mu \mathrm{m}$ longward of $3.0 \mu \mathrm{m}$. This behavior is consistent with the presence of amorphous $\mathrm{H}_{2} \mathrm{O}$ ice (Wada et al. 1991).

Our mean spectrum of the $3.0 \mu \mathrm{m}$ ice feature appears to be narrower than the one obtained by Chiar et al. (2002). In their model, the $3.0 \mu \mathrm{m}$ ice feature is best fitted by a temperature of $10 \mathrm{~K}$ and a maximum mantle thickness of 0.75 to $0.85 \mu \mathrm{m}$ assuming the simple model of coremantle grains of Bohren \& Huffman (1983) and the hypothesis of a variable mantle thickness of Smith et al. (1993).

- The $3.4 \mu \mathrm{m}$ and $3.48 \mu \mathrm{m}$ features are known to arise in the diffuse interstellar medium. They are both part of a single line complex (Duley \& Williams 1983) and have already been seen towards many Galactic Center sources (Sandford et al. 1991; Pendleton et al. 1994; Chiar et al. 2002; Mennella et al. 2003). They are most likely due to aliphatic hydrocarbons which are characterized by their $\mathrm{CH}_{2}$ (methylene) and $\mathrm{CH}_{3}$ (methyl) stretching modes (Sandford et al. 1991; Sellgren et al. 1995; Brooke et al. 1999; Grishko \& Duley 2002; Butchart et al. 1986; Duley \& Williams 1984).

Actually, the $3.4 \mu \mathrm{m}$ feature is composed of two bands, one at $3.38 \mu \mathrm{m}$ and the other at $3.42 \mu \mathrm{m}$. These are due to the asymmetric stretching vibrations of the $\mathrm{CH}_{3}$ and $\mathrm{CH}_{2}$ groups respectively (Sandford et al. 1991).

The $3.48 \mu \mathrm{m}$ seen in all spectra (see also the mean optical depth spectrum in Fig. 13) is due to the perturbed symmetric $\mathrm{C}-\mathrm{H}$ stretching vibrations of the same $\mathrm{CH}_{3}$ and $\mathrm{CH}_{2}$ groups (Sandford et al. 1991). This feature was already reported by Allen \& Wickramasinghe (1981), Jones et al. (1983) and Butchart et al. (1986).

The $3.4 \mu \mathrm{m}$ absorption features for the dusty sources and the He-stars are shown in Fig. 15. For both types of source the short wavelength wing is very similar. The long wavelength wing of the line feature derived from the He-stars is affected by the continuum mismatch discussed in Sect. 3.1. 
Table 2. The optical depths of the 4 absorption features at $3.0 \mu \mathrm{m}, 3.4 \mu \mathrm{m}$ and $3.48 \mu \mathrm{m}$.

\begin{tabular}{lcccc}
\hline \hline Object & $\tau_{3.0 \mu \mathrm{m}}$ & $\tau_{3.4 \mu \mathrm{m}}$ & $\tau_{3.4 \mu \mathrm{m}}$ lower limit & $\tau_{3.48 \mu \mathrm{m}}$ \\
\hline IRS13 & $0.44 \pm 0.03$ & $0.26 \pm 0.02$ & $0.16 \pm 0.02$ & $0.19 \pm 0.02$ \\
IRS13N & $0.35 \pm 0.04$ & $0.33 \pm 0.04$ & $0.21 \pm 0.03$ & $0.25 \pm 0.03$ \\
IRS16C & $0.67 \pm 0.04$ & $0.49 \pm 0.05$ & $0.14 \pm 0.02$ & $0.45 \pm 0.03$ \\
IRS16CC & $0.425 \pm 0.05$ & $0.36 \pm 0.03$ & $0.16 \pm 0.03$ & $0.31 \pm 0.02$ \\
IRS16NE & $0.38 \pm 0.05$ & $0.27 \pm 0.02$ & $0.14 \pm 0.02$ & $0.23 \pm 0.02$ \\
IRS16SW & $0.57 \pm 0.03$ & $0.36 \pm 0.05$ & $0.12 \pm 0.02$ & $0.29 \pm 0.03$ \\
IRS1W & $0.51 \pm 0.28$ & $0.32 \pm 0.04$ & $0.16 \pm 0.02$ & $0.27 \pm 0.02$ \\
IRS21 & $0.58 \pm 0.03$ & $0.23 \pm 0.04$ & $0.15 \pm 0.03$ & $0.16 \pm 0.02$ \\
IRS29 & $1.12 \pm 0.09$ & $0.37 \pm 0.03$ & $0.27 \pm 0.03$ & $0.29 \pm 0.02$ \\
IRS3 & $0.30 \pm 0.02$ & $0.23 \pm 0.03$ & $0.20 \pm 0.02$ & $0.15 \pm 0.01$ \\
IRS7 & $0.68 \pm 0.03$ & $0.41 \pm 0.06$ & $0.20 \pm 0.05$ & $0.33 \pm 0.04$ \\
IRS9 & $0.67 \pm 0.06$ & $0.27 \pm 0.07$ & $0.16 \pm 0.04$ & $0.19 \pm 0.03$ \\
\hline
\end{tabular}

\subsection{Measurements of the strengths of the absorption features}

The optical depths of the 4 absorption features at $3.0 \mu \mathrm{m}$, $3.4 \mu \mathrm{m}$ and $3.48 \mu \mathrm{m}$ are listed in Table 2 . The values are obtained by averaging the optical depth spectra over the wavelength intervals ]2.95 $\mu \mathrm{m}, 3.05 \mu \mathrm{m}[] ,3.35 \mu \mathrm{m}, 3.45 \mu \mathrm{m}[$ (thus including the two bands of the feature) and $] 3.43 \mu \mathrm{m}, 3.53 \mu \mathrm{m}[$, respectively. The $3.4 \mu \mathrm{m}$ and $3.48 \mu \mathrm{m}$ optical depths are measured in the spectra from which the $3 \mu \mathrm{m}$ ice absorption feature was subtracted (see Sect. 4.1).

The error bars listed in the table take into account only the measuring uncertainties, except for the $3.0 \mu \mathrm{m}$ feature of IRS $1 \mathrm{~W}$ where the error bar is taken such that the optical depth value includes the upper and lower limits obtained by fitting upper and lower baselines as described in Sect. 3.1.2.

To estimate the lower limit of the optical depth values of the $3.4 \mu \mathrm{m}$ absorption feature, we measured the value of the strength of this feature using a linear continuum between $3.25 \mu \mathrm{m}$ and $3.65 \mu \mathrm{m}$ as baseline. The obtained values are listed in Table 2.

Table 2 shows that the optical depth values span a large interval, suggesting that part of the absorption features arises probably from the local medium and may be associated with the individual sources.

Concerning the optical depth values of the $3.0 \mu \mathrm{m}$ feature, they agree well with the values obtained by Chiar et al. (2002) for the two sources in common, IRS 1W and IRS 7. The $3.4 \mu \mathrm{m}$ feature of these two sources exhibits higher values than those of Chiar et al. (2002). This is probably the result of the derivation of the isolated ice absorption feature described in Sect. 4.1 which is sensitive to the choice of the subtracted spectrum (here the spectrum of IRS 29). In particular, in Chiar et al. (2002) the authors find a negative optical depth value at $3.3 \mu \mathrm{m}$ for IRS 7 due to the shape of the ice feature which is narrower than the mean ice absorption feature. Moreover, their optical depth value of the $3.4 \mu \mathrm{m}$ feature of IRS 7 is even smaller than the lower limit obtained in our spectrum.

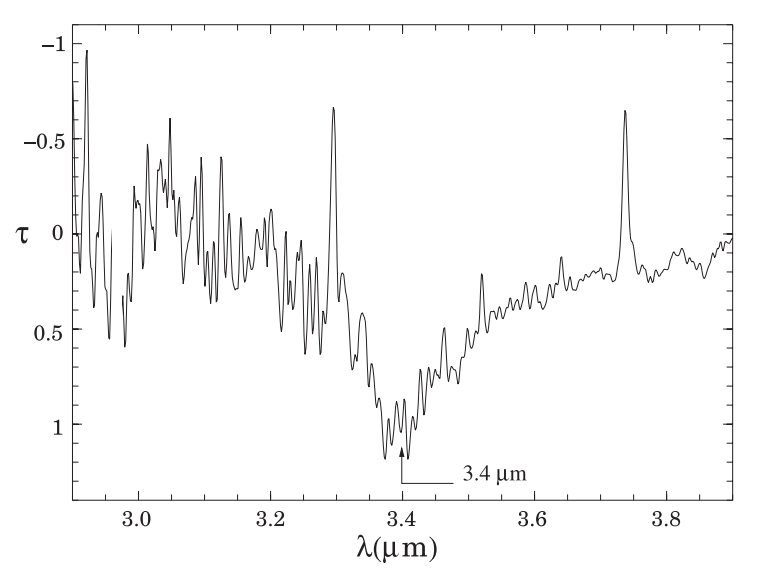

Fig. 16. Mean isolated and normalized $3.4 \mu \mathrm{m}$ feature of the dusty sources.

Concerning the IRS 3 source results, no comparison can be done with Chiar et al. (2002) as the fit of their spectrum was not satisfactory and did not match at all the $K$-band point flux. This may be due to the problematic relative flux calibration of their $L$-band spectrum which shows a different slope than ours.

On the other hand, the lower limits of the optical depth values of the hydrocarbon feature for the IRS 3 and IRS 7 sources in common with Sandford et al. (1991, 1995) and Pendelton et al. (1994) agree well with the values obtained by these authors. This is very satifactory as the values provided in these papers were derived using a linear fit similar to the one used here to derive the lower limits.

\subsection{Correlations between the strengths of the absorption features}

The plots of the optical depths of the absorption features at $3.4 \mu \mathrm{m}$ versus $3.0 \mu \mathrm{m}$ and $3.4 \mu \mathrm{m}$ versus $3.48 \mu \mathrm{m}$ are shown in Figs. 19 and 18 respectively. In these figures we also plot 
the data by Chiar et al. (2001) that were mostly obtained for sources in the outer parts of the central stellar cluster.

We find a good correlation (correlation coefficient of $\sim 0.98$ ) between the $3.4 \mu \mathrm{m}$ and $3.48 \mu \mathrm{m}$ absorption features (Fig. 18) and a vague of correlation (correlation coefficient of $\sim 0.43$ ) between the $3.4 \mu \mathrm{m}$ and $3.0 \mu \mathrm{m}$ features (Fig. 19). In Figs. 18 and 19, the linear regressions are drawn as well; the goodness of fit probabilities are respectively 0.9996 and $8 \times 10^{-4}$. The first correlation is to be expected since both features (at $3.4 \mu \mathrm{m}$ and $3.48 \mu \mathrm{m}$ ) arise from the same functional groups, as explained in Sect. 4.2.

The possible correlation between the optical depths of the $3.4 \mu \mathrm{m}$ and $3.0 \mu \mathrm{m}$ features shown in Fig. 19 suggests that the ISM in the central region is a mixture of diffuse and dense material. On the other hand, if this correlation is real, then in this plot the discrepancy between the positions of IRS 7 derived by us and by Chiar et al. (2002) cannot be due only to the variability of the source. In the case of variability, both positions should follow the overall trend of the correlation. Actually, the discrepancy between the positions of the 3 sources (IRS $1 \mathrm{~W}$, IRS 3 and IRS 7) in common with Chiar et al. (2002) is due to the same reasons mentioned in Sect. 4.3.

The fact that the $3.4 \mu \mathrm{m}$ to $3.0 \mu \mathrm{m}$ absorption feature "correlation" shows a significant offset from the origin of the coordinate system used for display may indicate that there could be significant $3.4 \mu \mathrm{m}$ line absorption even if the water ice feature were not present. Therefore we may conclude that a certain amount of the $3.4 \mu \mathrm{m}$ line absorption is due to the diffuse ISM on the line of sight to the Galactic Center. Due to the low density of the mini-spiral gas a substantial amount may, in fact, be closely linked to the individual sources that we are studying here in detail. Therefore, the remaining portion of the $3.4 \mu \mathrm{m}$ absorption may occur in the central parsec. This is also supported by the finding of Sandford et al. (1995) who derived anomalously high aliphatic $\mathrm{CH}$ absorption with respect to visual extinction relative to all the other lines of sight for which data were then available.

\subsection{Hydrogen emission lines}

The obtained $L$-band spectra also allow us to investigate the HI line emission towards selected lines of sight. The corresponding line strengths, widths and equivalent widths for $\operatorname{Br} \alpha$, $\operatorname{Pf} \gamma$, and $\operatorname{Pf} \delta$ are listed in Tables 4-6. At the achieved spectral resolution we cannot distinguish between emission from the individual objects and from the mini-spiral. The latter seems to dominate the emission since sources in or close to the minispiral (IRS 1W, 16NE, 16SW, 21, 13, 13N) also show the strongest line emission. Especially for IRS 21 the $K$-band spectrum by Ott et al. (1999) suggests that probably all the hydrogen line emission is due to the mini-spiral.

Using the $\mathrm{Br}_{\gamma}$ and $\mathrm{P}_{\alpha}$ line strengths published by Najarro et al. (1997) we can calculate the diagnostic line ratios $\mathrm{Br}_{\gamma} / \mathrm{Br}_{\alpha}$ and $\mathrm{P}_{\alpha} / \mathrm{Br}_{\alpha}$ for three of the IRS 16 sources. The results are shown in Table 3. To obtain dereddened line strengths we have considered an absorption in the $L$-band wavelength range of $A_{L}=1.68$, consistent with our optical depth spectrum

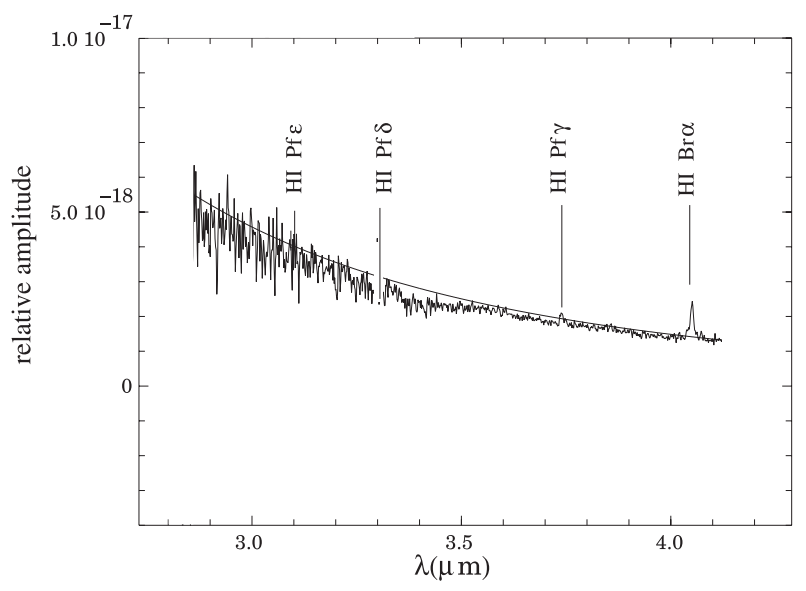

Fig. 17. $L$-band intrinsic spectrum (i.e. corrected for extinction along the line of sight as explained in Sect. 3.2) at the position of SgrA* taken through a $0.6^{\prime \prime}$ wide slit using ISAAC on the VLT UT1. The continuum of a blackbody of $22200 \mathrm{~K}$ is also shown. The locations of hydrogen lines are marked.

of Fig. 8 as well as with the value given by Rieke \& Lebofsky (1985).

Taking the dereddened ratios at face value and omitting possible small contributions from the He line emission close to the $\mathrm{P} \alpha$ and $\mathrm{Br} \gamma$ line we can derive first estimates for the temperature and density of the emitting gas. Assuming case B recombination, for both sources IRS 16NE and IRS 16C the line ratios are consistent with emission from gas at low temperatures of $\leq 5000 \mathrm{~K}$ and low densities of $\leq 10^{2} \mathrm{~cm}^{-3}$. For IRS $16 \mathrm{SW}$ the ratios are more consistent with emission from a gas at high temperature $\sim 20000 \mathrm{~K}$ and high density $\sim 10^{4} \mathrm{~cm}^{-3}$. While it cannot be excluded that the emission towards IRS 16SW contains a significant contribution from the hot stellar atmospheres of the He-star IRS 16SW and the other neighboring hot stars in the IRS 16 complex, this result reflects the complex density and temperature structure within the mini-spiral, which consists of a thermal plasma of $n_{\mathrm{e}} \sim 10^{4} \mathrm{~cm}^{-3}$ at $T \sim 10^{4} \mathrm{~K}$ (e.g. Brown et al. 1981) with denser regions of $n_{\mathrm{H}}>10^{5} \mathrm{~cm}^{-3}$ and a few $100 \mathrm{~K}$ (e.g. Jackson et al. 1993).

\subsection{L-band spectrum at the position of SgrA*}

Our ISAAC $L$-band imaging and spectroscopy was part of a flux density monitoring program of SgrA* (Baganoff et al. 2001, 2003; Eckart et al. 2004) which has been carried out simultaneously with CHANDRA observations. Therefore all slit settings were defined such that $\operatorname{SgrA}^{*}$ always fell within the slit. A mean extinction corrected spectrum at that position is shown in Fig. 17. Here the weak hydrogen recombination lines are most likely due to emission from the mini-spiral. The Rayleigh-Jeans shape of the continuum spectrum is well comparable to that of other hot stars, like the He stars in the central cluster. In Fig. 17 we show the $L$-band spectrum of SgrA* compared with a $22200 \mathrm{~K}$ blackbody spectrum. In fact, high resolution adaptive optics $L$-band images were taken in August 2002 with NAOS/CONICA on the VLT UT4 during the science verification phase (Clénet et al. 2003; Genzel et al. 2003; 


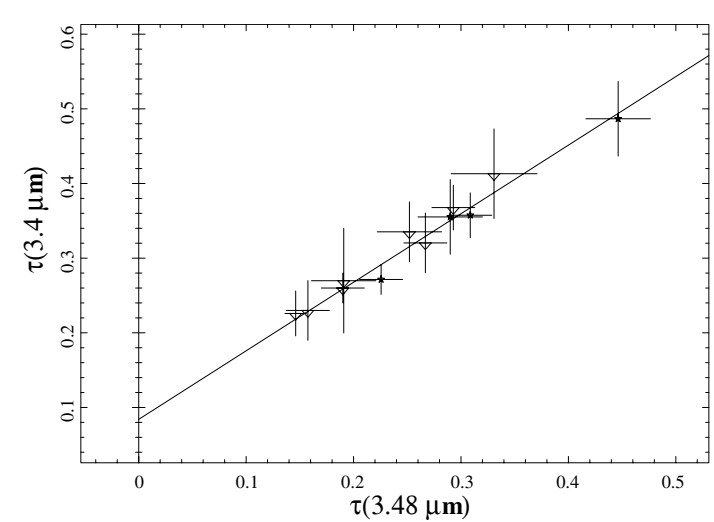

Fig. 18. Optical depth of the hydrocarbon absorption at the $3.4 \mu \mathrm{m}$ versus the $3.48 \mu \mathrm{m}$ absorption feature for all observed Galactic Center sources (the IRS 16 sources are represented by stars and the remaining sources by triangles).

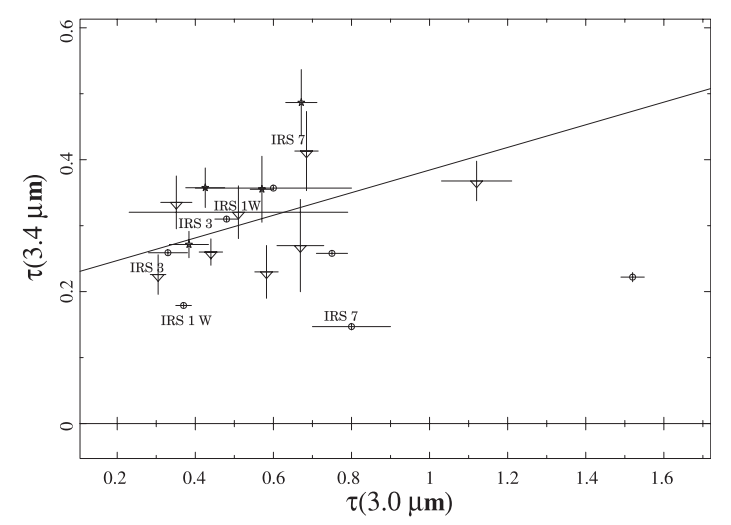

Fig. 19. Optical depth of the hydrocarbon absorption at $3.4 \mu \mathrm{m}$ versus the depth in the $3.0 \mu \mathrm{m}$ absorption feature for all observed Galactic Center sources (the IRS 16 sources are represented by stars and the remaining sources by triangles). The values obtained by Chiar et al. (2002) are also shown (in circles) but are not considered in the linear regression fit.

Table 3. Diagnostic line ratios for the three IRS 16 sources in common with Najarro et al. (1997).

\begin{tabular}{lcc}
\hline \hline Object & $\left(\mathrm{P}_{\alpha}+\mathrm{He}\right) / \mathrm{Br}_{\alpha}$ & $\left(\mathrm{Br}_{\gamma}+\mathrm{He}\right) / \mathrm{Br}_{\alpha}$ \\
\hline IRS16NE & $3.2 \pm 0.6$ & $0.28 \pm 0.03$ \\
IRS16C & $3.4 \pm 0.6$ & $0.26 \pm 0.03$ \\
IRS16SW & $4.6 \pm 0.8$ & $0.43 \pm 0.04$ \\
\hline
\end{tabular}

Eckart et al. 2004). These images show that at the position of SgrA* the $L$-band flux density is dominated by the fast moving star S2 (Schödel et al. 2003). Our spectrum is consistent with the fact that based on its $K$-band luminosity spectrum $\mathrm{S} 2$ is likely to be a $\sim 15-20$ solar mass late O, early B main-sequence star of age less than $20 \mathrm{Myr}$ (Gezari et al. 2002; Eckart et al. 1999; Figer et al. 2001; Ghez et al. 2003).

\section{IRS 13N}

Well within the central stellar cluster of the Milky Way, about $0.5^{\prime \prime}$ north of the IRS 13 complex, Eckart et al. (2004) found a small cluster $(<0.13$ light years) of compact sources with a strong infrared excess and obtained a first spectrum of it. The integrated spectrum of that area clearly shows that this excess is due to the contribution of warm $T=500-1000 \mathrm{~K}$ dust. The nature of these newly found sources is currently unclear. Eckart et al. (2004) discuss three possible explanations. The $L$-band excess sources north of the IRS 13 complex could be heavily extincted luminous stars. They could also be hot stars that heat and interact with the environment of the local mini-spiral. Finally they may even be young stars and their $L$-band excess may be due to the flux density contribution of luminous accretion disks. While a combination of the first two possibilities currently cannot be entirely excluded, the luminosities and colours of these sources are consistent with those of young stellar objects. As described in Sect. 3.1, a reddened blackbody continuum was also fitted to the IRS $13 \mathrm{~N}$ spectrum considering the $K$ - and $L$-band fluxes given by Eckart et al. (2004) (see Figs. 2 and 9 and Table 1). The resulting temperature and $K$-band extinction agree well with the above explanations. The extinction of about $3.9 \mathrm{mag}$ in the $K$-band is about 1 mag higher than the overall line of sight extinction toward the Galactic Center, and a $\sim 1000 \mathrm{~K}$ dust temperature emission agrees well with the highly dust embedded YSOs as studied by e.g. Ishii et al. (1998). The ice absorption feature often observed towards YSOs (see Ishii et al. 1998) is not detectable in the extinction corrected spectrum shown in Fig. 9; this may in part be due to the small absorption in the spectrum of the late-type CO-star that is reflected in the spectrum of the extinction along the line of sight (Fig. 8). It is also possible that the circumstellar material is affected by the intense radiation field at the Galactic Center (Lutz et al. 1996). In this case the ices in the disks of young stars may either become tenuous or be destroyed by hard UV photons.

\section{Summary and conclusions}

Combined NIR and MIR spectroscopic observations of sources in the central $0.5 \mathrm{pc}$ parsec of the Milky Way allowed us to obtain a detailed picture of the absorption features visible in the spectra towards that region. Our investigation of the central sources (radius $\leq 2.5^{\prime \prime}$ ) complements the study by Chiar et al. (2002) which mostly includes sources with larger separations from the center. We find some evidence that the diffuse ISM in the central $0.5 \mathrm{pc}$ has properties that are slightly distinct from the ISM at larger distances from the center.

The $3.0 \mu \mathrm{m}$ ice profile usually observed toward the Galactic Center peaks at $2.96 \mu \mathrm{m}$, shortward of the ice feature in local molecular clouds (McFadzean et al. 1989; Tielens et al. 1996; Chiar et al. 2000). It is likely due to cold (15 K) water ices with an enhanced $\mathrm{NH}_{3}$ abundance (Chiar et al. 2000). Especially processed ices contribute a substantial portion of the refractory grain materials that persist when the molecular cloud is dispersed by star formation, and these products may yield the extinction characteristic of the diffuse interstellar medium.

However, the profile of the $3.0 \mu \mathrm{m}$ ice feature obtained in our work peaks longward of $3.0 \mu \mathrm{m}$ and is therefore likely to be associated with the presence of amorphous $\mathrm{H}_{2} \mathrm{O}$ ice towards these sources. 
Simultaneous fits of our $K$ - and $L$-band spectra with single reddened blackbody continua allowed us to estimate the extinction towards the individual Galactic Center sources and to determine the approximate continuum shape of the observed spectra. The derived $K$-band extinctions and blackbody temperatures are consistent with values found for the sources in the central stellar cluster (Chiar et al. 2002; Tanner et al. 2002, 2003).

Using the spectrum of a late-type star assumed to be free from local extinction, we were able to derive the spectrum of the $L$-band extinction along the line of sight toward the Galactic Center. This spectrum has been used to derive the extinction corrected spectra of the most luminous sources. The extinction corrected spectra are consistent with the blackbody temperatures derived from the previous fitting procedure. The Rayleigh-Jeans continuum spectra obtained towards all the hot stars indicate that the distribution of the extinction in the central half parsec is fairly flat and varies in the $K$-band only by $\Delta A_{K} \sim \pm 0.5 \mathrm{mag}$. This is consistent with the results of Scoville et al. (2003). Therefore the excess extinction that we determined after correction for the line of sight extinction towards some of the sources should be associated with the individual objects or very clumpy features of the ISM. This is in support of the findings by Blum et al. (1996) and Clénet et al. (2001) and is also consistent with the large range of the optical depth values derived from the fitting procedure.

The presence of local extinction in the envelopes of dusty sources is consistent with the bow shock model of Tanner et al. (2002, 2003). Cotera et al. (1999) had already shown that several of these sources are indeed offset from nearby local maxima in the extended dust emission and temperature distribution. Especially for IRS 21, Tanner et al. (2002) indicate that the extended dust emission of this source is consistent with a bow shock created by the motion of such a massive hot star through the dust and gas of the mini-spiral. It is likely that the bow shock scenario may be applicable to most of the dust-embedded sources in the central stellar cluster. For IRS 3, however, the bow-shock scenario may not apply. Gezari et al. (1985) find that IRS 3 is the most compact and (together with IRS 7) hottest bright source ( $T \sim 400 \mathrm{~K})$ in the central cluster. As suggested in the case of IRS 21 by Tanner et al. (2002) IRS 3 may be an optically thick dust shell surrounding a mass-losing source, such as a dusty recently formed WC9 Wolf-Rayet star.

In addition, a blackbody temperature of $\sim 1000 \mathrm{~K}$ is found for the spectrum representing the highly reddened sources located to the North of IRS 13. While it cannot be excluded that the individual objects contained in this source complex are lower luminosity analogues of the class of bow shock objects found by Tanner et al. (2002) and Rigaut et al. (2003), their temperature and luminosity is in good agreement with the low temperatures of the YSOs classified by Ishii et al. (1998).

Detailed modeling, similar to the studies by Tanner et al. (2003) and Eckart et al. (2004), based on higher angular resolution MIR imaging and spectroscopy (using AO or interferometry) is required to unravel the nature of the highly extincted sources in the IRS $13 \mathrm{~N}$ association.
Finally, a vague correlation is noticed in the $3.4 \mu \mathrm{m}$ versus $3.0 \mu \mathrm{m}$ optical depths plot. If a real correlation were confirmed between these two features, it would suggest that the ISM along the line of sight toward the Galactic Center is possibly composed of a mixture of diffuse and dense material. Moreover, the plots of the optical depth values suggests that part of the $3.4 \mu \mathrm{m}$ feature arises probably from the foreground ISM and part of it from the local medium associated with the individual sources.

Acknowledgements. This work was supported in part by the Deutsche Forschungsgemeinschaft (DFG) via grant SFB 494. We are grateful to all members of the ISAAC/VLT and the MPE 3D team.

\section{References}

Allen, D. A., \& Wickramasinghe, D. T. 1981, Nature, 294, 239

Baganoff, F. K., Bautz, M. W., Brandt, W. N., et al. 2001, Nature, 413, 45

Baganoff, F. K., et al. 2003, Proc. of the Galactic Center Workshop, Nov. 3-8, 2002, Hawaii, ed. A. Cotera, T. Geballe, S. Markoff, \& H. Falcke, Astron. Nachr., in press

Becklin, E. E., \& Neugebauer, G. 1968, iih Conf., 1

Becklin, E. E., \& Neugebauer, G. 1969, ApJ, 157, L31

Becklin, E. E., \& Neugebauer, G. 1975, ApJ, 200, L71

Becklin, E. E., Matthews, K., Neugebauer, G., \& Willner, S. P. 1978, ApJ, 219, 121

Blum, R. D., Sellgren, K., \& Depoy, D. L. 1988, AJ, 112

Blum, R. D., Sellgren, K., \& Depoy, D. L. 1995a, ApJ, 440, L17

Blum, R. D., Depoy, D. L., \& Sellgren, K. 1995b, ApJ, 441, 603

Blum, R. D., Sellgren, K., \& Depoy, D. L. 1996, ApJ, 470, 864

Bohren, C. F., \& Huffman, D. R. 1983, asls. book

Brandner, W., et al. 2002, The ESO Messenger, 107, 1

Brooke, T. Y., Sellgren, K., \& Geballe, T. R. 1999, ApJ, 517, 883

Brooke, T. Y., Tokunaga, A. T., \& Strom, S. E. 1993, AJ, 106, 656

Brown, R. L., Johnston, K. J., \& Lo, K. Y. 1981, ApJ, 250, 155

Butchart, I., McFadzean, A. D., Whittet, D. C. B., Geballe, T. R., \& Greenberg, J. M. 1986, A\&A, 154

Chan, Kin-Wing, Moseley, S. H., Casey, S., et al. 1997, ApJ, 483, 798

Chiar, J. E., Tielens, A. G. G. M., Whittet, D. C. B., et al. 2000, ApJ, 537, 749

Chiar, J. E., \& Tielens, A. G. G. M. 2001, ApJ, 550, L207

Chiar, J. E., Adamson, A. J., Pendleton, Y. J., et al. 2002, ApJ, 570, 198

Clénet, Y., Rouan, D., Gendron, E., et al. 2001, A\&A, 376, 124

Clénet, Y., et al. 2003, Proc. of the Galactic Center Workshop, Nov. 3-8, 2002, Hawaii, ed. A. Cotera, T. Geballe, S. Markoff, \& H. Falcke, Astron. Nachr., in press

Cotera, A. S., Erickson, E. F., Simpson, J. P., \& Rieke, M. 1992, J. Amer. Astron. Soc., 180th A\&AS Meet., 25.02, BAAS, 24, 765

Cotera, A. S., Erickson, E. F., Colgan, S. W. J., et al. 1996, ApJ, 461, 750

Cotera, A. S., Simpson, J. P., Erickson, E. F., et al. 1999a, ApJ, 510, 747

Cotera, A., Morris, M., Ghez, A. M., et al. 1999b, cpg Conf., 240

de Graauw, T., Whittet, D. C. B., Gerakines, P. A., et al. 1996, A\&A, 315, L345

Duley, W. W., \& Williams, D. A. 1983, MNRAS, 205, 67

Duley, W. W., \& Williams, D. A. 1984, Nature, 311, 685

Eckart, A., \& Genzel, R. 1996, Nature, 383, 415

Eckart, A., Genzel, R., Hofmann, R., Sams, B. J., \& Tacconi-Garman, L. E. 1995, ApJ, 445, L26 
Eckart, A., Ott, T., Genzel, R., \& Lutz, D. 1998, in Proc. IAU Symp., No. 193 on Wolf-Rayet Phenomena in Massive Stars and Starburst Galaxies, Puetrto Valarta, Mexico, November 3-7, ed. K. A. van der Hucht, G. Koenigsberger, \& P. R. J. Enens (Kluewer), 449

Eckart, A., Ott, T., \& Genzel, R. 1999, A\&A, 352, L22

Eckart, A., Genzel, R., Ott, T., \& Schoedel, R. 2002, MNRAS, 331, 917

Eckart, A., Moultaka, J., et al. 2003, Proc. of the Galactic Center Workshop, Nov. 3-8, 2002, Hawaii, ed. A. Cotera, T. Geballe, S. Markoff, \& H. Falcke, Astron. Nachr., in press

Eckart, A., Moultaka, J., Viehmann, T., Straubmeier, C., \& Mouawad, N. 2004, ApJ, 602, in press

Eisenhauer, F., Schoedel, R., Genzel, R., et al., ApJL, accepted [arXiv: astro-ph/0306220]

Figer, D. F., Najarro, F., McLean, I. S., Morris, M., \& Geballe, Th. R. 1997, Luminous Blue Variables: Massive Stars in Transition, ed. A. Nota, \& H. Lamers, ASP Conf. Ser., 120, 196

Figer, D. F., Gilmore, D., Morris, M., et al. 2001, A\&AS, 198, 8706

Figer, D. F., Najarro, F., Gilmore, D., et al. 2002, ApJ, 581, 258

Fuente, A., Martin-Pintado, J., Bachiller, R., Rodriguez-Franco, A., \& Palla, F. 2002, A\&A, 387, 977

Genzel, R., Thatte, N., Krabbe, A., Kroker, H., \& Tacconi-Garman, L. E. 1996, ApJ, 472, 153

Genzel, G., Eckart, A., Ott, T., \& Eisenhauer, F. 1997, MNRAS, 291, 219

Genzel, R., Pichon, C., Eckart, A., Gerhard, O., \& Ott, T. 2000, MNRAS, 317, 348

Genzel, R., Schödel, R., Ott, T., et al. 2003a, Nature, 425, 934

Genzel, R., Schödel, R., Ott, T., et al. 2003b, ApJ, 594, 812

Gerakines, P. A., Whittet, D. C. B., Ehrenfreund, P., et al. 1999a, ApJ, 522,357

Gerakines, P. A., Whittet, D. C. B., Ehrenfreund, P., et al. 1999b, ApJ, 526,1062

Gerhard, O. 2001, ApJ, 546, L39

Gezari, D. Y., Shu, P., Lamb, G., et al. 1985, ApJ, 299, 1007

Gezari, D. Y., Schmitz, M., Pitts, P. S., \& Mead, J. M. 1993, Catalogue of Infrared Observations (NASA RP-1294), 3rd ed. (Washington: NASA)

Gezari, D., Dwek, E., \& Varosi, F. 1994, in The Nuclei of Normal Galaxies: Lessons from the Galactic Center, Proc. of the NATO Advanced Research Workshop, held in Schloss Ringberg, Kreuth, Bavaria, Germany, July 25-30, 1993 (Dordrecht: Kluwer Academic Publishers), ed. R. Genzel, \& A. I. Harris, NATO Advanced Science Institutes (ASI) Series C, 445, 343

Gezari, D., Dwek, E., \& Varosi, F. 1996, IAUS, 169, 231

Gezari, S., Ghez, A. M., Becklin, E. E., et al. 2002, ApJ, 576, 790

Ghez, A., Klein, B. L., Morris, M., \& Becklin, E. E. 1998, ApJ, 509, 678

Ghez, A., Morris, M., Becklin, E. E., Tanner, A., \& Kremenek, T. 2000, Nature, 407, 349

Ghez, A., Duchêne, G., Matthews, K., et al. 2003, ApJ, 586, L127

Gillett, F. C., \& Forrest, W. J. 1973, ApJ, 179, 483

Glass, I. S., \& Moorwood, A. F. M. 1985, MNRAS, 214, 429

Grishko, V. I., \& Duley, W. W. 2002, ApJ, 568, L131

Guesten, R., Genzel, R., Wright, M. C. H., et al. 1987, ApJ, 318, 124

Hagen, W., Greenberg, J. M., \& Tielens, A. G. G. M. 1983, A\&A, 117, 132

Herbst, T. M., Beckwith, S. V. W., \& Shure, M. 1993, ApJ, 411, L21

Hillenbrand, L. A., Strom, S. E., Vrba, F. J., \& Keene, J. 1992, ApJ, 397,613

Hoyle, F., Wickramasinghe, N. C., Al-Mufti, S., Olavesen, A. H., \& Wickramasinghe, D. T. 1982, Ap\&SS, 83, 405
Hudgins, D. M., Sandford, S. A., Allamandola, L. J., \& Tielens, A. G. G. M. 1993, ApJS, 86, 713

Ishii, M., Nagata, T., Sato, S., et al. 1998, AJ, 116, 868

Jackson, J. M., Geis, N., Genzel, R., et al. 1993, ApJ, 402, 173

Jones, T. J., Hyland, A. R., \& Allen, D. A. 1983, MNRAS, 205, 187

Joyce, R. R., \& Simon, T. 1982, ApJ, 260, 604

Kim, S. S., Morris, M., \& Lee, H. M. 1999, ApJ, 525, 228

Kitta, K., \& Kraetschmer, W. 1983, A\&A, 122, 105

Kleinmann, S. G., \& Hall, D. N. B. 1986, ApJS, 62, 501

Koornneef, J. 1983, A\&A, 128, 84

Krabbe, A., Genzel, R., Eckart, A., et al. 1995, ApJ, 447, L95

Lacy, J. H., Townes, C. H., \& Hollenbach, D. J. 1982, ApJ, 262, 120

Lebofsky, M. J. 1979, AJ, 84, 324

Lenzen, R., Hofmann, R., Bizenberger, P., \& Tusche, A. 1998, Proc. SPIE, IR Astronomical Instrum, ed. A. M. Fowle, 3354, 606

Lutz, D., Feuchtgruber, H., Genzel, R., et al. 1996, A\&A, 315, 269

Maillard, J. P., et al. 2003, Proc. of the Galactic Center Workshop, Nov. 3-8, 2002, Hawaii, ed. A. Cotera, T. Geballe, S. Markoff, \& H. Falcke, Astron. Nachr., in press

Maldoni, M. M., Smith, R. G., Robinson, G., \& Rookyard, V. L. 1998, MNRAS, 298, 251

Martin, P. G., \& Whittet, D. C. B. 1990, ApJ, 357, 113

McFadzean, A. D., Whittet, D. C. B., Bode, M. F., Adamson, A. J., \& Longmore, A. J. 1989, MNRAS, 241, 873

Mennella, V., Baratta, G. A., Esposito, A., Ferini, G., \& Pendleton, Y. J. 2003, ApJ, 587, 727

Moneti, A., Cernicharo, J., \& Pardo, J. R. 2001a, ApJ, 549, L203

Morris, M. 1993, ApJ, 408, 496

Najarro, F., Krabbe, A., Genzel, R., et al. 1997, A\&A, 325, 700

Ott, T., Eckart, A., \& Genzel, R. 2003, ApJ, 523, 2480

Paumard, T., Maillard, J. P., Morris, M., \& Rigaut, F. 2001, A\&A, 366, 466

Pendleton, Y. J., Sandford, S. A., Allamandola, L. J., Tielens, A. G. G. M., \& Sellgren, K. 1994, ApJ, 437, 683

Phinney, E. S. 1989, in The Centre of the Galaxy, ed. M. Morris (Dortrecht: Kluwer), 543

Portegies, Z. S., McMillan, S., \& Gerhard, O. 2003 [arXiv: astro-ph/0303599], ApJ, in press

Reid, M. J., Menten, K. M., Genzel, R. A., et al. 2003, ApJ, 587, 208

Rieke, G. H., \& Low, F. G. 1973, ApJ, 184, 415

Rieke, G. H., \& Lebofsky, M. J. 1985, ApJ, 288, 618

Rieke, G. H., Rieke, M. J., \& Paul, A. E. 1989, ApJ, 336, 752

Rigaut, et al. 2003, Proceedings of the Galactic Center Workshop, Nov. 3-8, 2002, Hawaii, ed. A. Cotera, T. Geballe, S. Markoff, $\&$ H. Falcke, Astron. Nachr., in press

Rousset, G., et al. 1998, Proc. SPIE Adaptive Optics Technology, ed. D. Bonaccini, \& R. K. Tyson, 3353, 508

Sanders, R. H. 1992, Nature, 359, 131

Sandford, S. A., Allamandola, L. J., Tielens, A. G. G. M., et al. 1991, ApJ, 371, 607

Sandford, S. A., Pendleton, Y. J., \& Allamandola, L. J. 1995, ApJ, 440,697

Scoville, N. Z., Stolovy, S. R., Rieke, M., Christopher, M. H., \& Yusef-Zadeh, F. 2003, ApJ, accepted

Schödel, R., Ott, T., Genzel, R., et al. 2002, Nature, 419, 694

Schödel, R., Ott, T., Genzel, R., et al. 2003, ApJ, 596, 1015

Sellgren, K., McGinn, M. T., Becklin, E. E., \& Hall, D. N. 1990, ApJ, 359,112

Sellgren, K., Brooke, T. Y., Smith, R. G., \& Geballe, T. R. 1995, ApJ, 449, L69

Serabyn, E., \& Morris, M. 1996, Nature, 382, 602

Simon, M., Chen, W. J., Forrest, W. J., et al. 1990, ApJ, 360, 95

Smith, R. G., Sellgren, K., \& Brooke, T. Y. 1993, MNRAS, 263, 749 
Storey, J. W. V., \& Allen, D. A. 1983, MNRAS, 204, 1153

Tamura, M., Werner, M. W., Becklin, E. E., \& Phinney, E. S. 1994, iaan Conf., 117

Tamura, M., Werner, M. W., Becklin, E. E., \& Phinney, E. S. 1996, ApJ, 467, 645

Tanner, A., Ghez, A. M., Morris, M., et al. 2002, ApJ, 575, 860

Tanner, et al. 2003, Proceedings of the Galactic Center Workshop, Nov. 3-8, 2002, Hawaii, ed. A. Cotera, T. Geballe, S. Markoff, $\&$ H. Falcke, Astron. Nachr., in press

Thatte, N. A., Kroker, H., Weitzel, L., et al. 1995, SPIE., 2475, 228

Thornley, M. D., Schreiber, N. M. F., Lutz, D., et al. 2000, ApJ, 539, 641

Tielens, A. G. G. M., \& Hagen, W. 1982, A\&A, 114, 245
Tielens, A. G. G. M., Hagen, W., \& Greenberg, J. M. 1983, JPhCh, 87,4220

Tielens, A. G. G. M., Wooden, D. H., Allamandola, L. J., Bregman, J., \& Witteborn, F. C. 1996, ApJ, 461, 210

Tokunaga, A. T. 1997, in Diffuse Infrared Radiation and the IRTS, ed. H. Okuda, T. Matsumoto, \& T. Rollig, ASP Conf. Ser., 124, 149 Tollestrup, E. V., Becklin, E. E., \& Capps, R. W. 1989, AJ, 98, 204

Wada, S., Sakata, A., \& Tokunaga, A. T. 1991, ApJ, 375, L17

Weitzel, L., Krabbe, A., Kroker, H., et al. 1996, A\&AS, 119, 531

Willner, S. P., Gillet, F. C., Herter, T. L., et al. 1982, ApJ, 253, 174

Yusef-Zadeh, F., Roberts, D. A., \& Biretta, J. 1998, ApJ, 499, L159

Zhao, J.-H., \& Goss, W. M. 1998, ApJ, 499, L163 
J. Moultaka et al.: Interstellar matter and circumstellar matter in the central parsec of the Milky Way, Online Material p 1

\section{Online Material}


J. Moultaka et al.: Interstellar matter and circumstellar matter in the central parsec of the Milky Way, Online Material p 2

Table 4. Continuum and emission line strengths as well as linewidths and equivalent widths of the $\operatorname{Br} \alpha$ line towards the observed sources.

\begin{tabular}{lcccc}
\hline \hline Object & Line-continuum of HI $(4.051 \mu \mathrm{m})$ & Integral of HI $(4.051 \mu \mathrm{m})$ & $E W$ of HI $(4.051 \mu \mathrm{m})$ & \multicolumn{2}{c}{ Line width } \\
& $10^{-18} \mathrm{~W} \mathrm{~cm}^{-2} \mu \mathrm{m}^{-1}$ & $10^{-18} \mathrm{~W} \mathrm{~cm}^{-2}$ & $\AA$ & $\AA$ \\
\hline IRS 1 W & $55.9 \pm 0.40$ & $0.5869 \pm 0.1216$ & $-41.48 \pm 1.86$ & $210 \pm 42$ \\
IRS 3 & $5.80 \pm 0.50$ & $0.0855 \pm 0.0378$ & $-6.02 \pm 2.83$ & $295 \pm 105$ \\
IRS 7 & - & - & - & - \\
IRS 9 & $19.60 \pm 0.40$ & $0.1137 \pm 0.0121$ & $-63.56 \pm 1.64$ & $116 \pm 10$ \\
IRS 21 & $13.00 \pm 0.20$ & $0.1566 \pm 0.0362$ & $-48.03 \pm 1.90$ & $241 \pm 52$ \\
IRS 29 & $1.70 \pm 0.20$ & $0.0196 \pm 0.0041$ & $-10.26 \pm 1.75$ & $231 \pm 21$ \\
IRS 13 & $47.50 \pm 0.40$ & $0.8479 \pm 0.0570$ & $-87.59 \pm 2.63$ & $357 \pm 21$ \\
IRS 13 N & $35.60 \pm 0.30$ & $0.2243 \pm 0.0393$ & $-63.58 \pm 1.18$ & $126 \pm 21$ \\
IRS 16 CC & $1.69 \pm 0.03$ & $0.0275 \pm 0.0066$ & $-58.20 \pm 4.39$ & $326 \pm 73$ \\
IRS 16 C & $3.86 \pm 0.06$ & $0.0588 \pm 0.0028$ & $-117.43 \pm 3.28$ & $305 \pm 10$ \\
IRS 16 NE & $10.19 \pm 0.08$ & $0.1126 \pm 0.0065$ & $-112.82 \pm 2.94$ & $221 \pm 11$ \\
IRS 16 SW & $6.29 \pm 0.07$ & $0.1025 \pm 0.0109$ & $-120.66 \pm 3.61$ & $326 \pm 31$ \\
\hline
\end{tabular}

Table 5. Continuum and emission line strengths as well as linewidths and equivalent widths of the Pf $\gamma$ line towards the observed sources.

\begin{tabular}{lcccc}
\hline \hline Object & $\begin{array}{c}\text { Line-continuum of HI }(3.739 \mu \mathrm{m}) \\
10^{-18} \mathrm{~W} \mathrm{~cm}^{-2} \mu \mathrm{m}^{-1}\end{array}$ & $\begin{array}{c}\text { Integral of HI }(3.739 \mu \mathrm{m}) \\
10^{-18} \mathrm{~W} \mathrm{~cm}^{-2}\end{array}$ & $\begin{array}{c}\text { A of HI }(3.739 \mu \mathrm{m}) \\
\text { Line width }\end{array}$ \\
\hline IRS 1 W & $13.50 \pm 0.60$ & $0.1276 \pm 0.0198$ & $-12.86 \pm 1.36$ & $189 \pm 21$ \\
IRS 3 & $3.30 \pm 0.40$ & $0.0346 \pm 0.0077$ & $-4.79 \pm 1.06$ & $210 \pm 21$ \\
IRS 7 & $18.00 \pm 1.00$ & $0.1224 \pm 0.0158$ & $-4.41 \pm 0.52$ & $136 \pm 10$ \\
IRS 9 & $3.80 \pm 0.70$ & $0.0380 \pm 0.0091$ & $-12.63 \pm 1.67$ & $200 \pm 11$ \\
IRS 21 & $2.10 \pm 0.10$ & $0.0166 \pm 0.0041$ & $-9.29 \pm 0.69$ & $158 \pm 32$ \\
IRS 29 & $1.10 \pm 0.10$ & $0.0072 \pm 0.0009$ & $-3.73 \pm 0.31$ & $131 \pm 5$ \\
IRS 13 & $8.90 \pm 0.25$ & $0.0703 \pm 0.0064$ & $-15.29 \pm 0.86$ & $158 \pm 10$ \\
IRS 13 N & $6.30 \pm 0.30$ & $0.0627 \pm 0.0127$ & $-15.38 \pm 1.64$ & $199 \pm 31$ \\
IRS 16 CC & $0.37 \pm 0.03$ & $0.0039 \pm 0.0007$ & $-9.96 \pm 1.14$ & $210 \pm 21$ \\
IRS 16 C & $0.86 \pm 0.05$ & $0.0086 \pm 0.0009$ & $-20.03 \pm 1.84$ & $200 \pm 10$ \\
IRS 16 NE & $2.16 \pm 0.08$ & $0.0239 \pm 0.0042$ & $-21.74 \pm 2.50$ & $221 \pm 31$ \\
IRS 16 SW & $1.07 \pm 0.05$ & $0.0096 \pm 0.0010$ & $-19.72 \pm 1.53$ & $179 \pm 10$ \\
\hline
\end{tabular}


J. Moultaka et al.: Interstellar matter and circumstellar matter in the central parsec of the Milky Way, Online Material p 3

Table 6. Continuum and emission line strengths as well as linewidths and equivalent widths of the Pf $\delta$ line towards the observed sources.

\begin{tabular}{lcccc}
\hline \hline Object & Line-continuum of HI $(3.296 \mu \mathrm{m})$ & Integral of HI $(3.296 \mu \mathrm{m})$ & $E W$ of HI $(3.296 \mu \mathrm{m})$ & Line width \\
& $10^{-18} \mathrm{~W} \mathrm{~cm}^{-2} \mu \mathrm{m}^{-1}$ & $10^{-18} \mathrm{~W} \mathrm{~cm}^{-2}$ & $\AA$ & $\AA$ \\
\hline IRS 1 W & $7.80 \pm 0.20$ & $0.0573 \pm 0.0097$ & $-9.26 \pm 0.81$ & $147 \pm 21$ \\
IRS 3 & - & - & - & - \\
IRS 7 & $12.00 \pm 2.00$ & $0.0450 \pm 0.0135$ & $-3.50 \pm 0.94$ & $75 \pm 10$ \\
IRS 9 & - & - & - & - \\
IRS 21 & - & - & - & - \\
IRS 29 & - & - & - & - \\
IRS 13 & $4.80 \pm 0.20$ & $0.0226 \pm 0.0033$ & $-7.65 \pm 0.68$ & $94 \pm 10$ \\
IRS 13 N & $3.30 \pm 0.30$ & $0.0208 \pm 0.0054$ & $-8.29 \pm 0.99$ & $126 \pm 21$ \\
IRS 16 CC & - & - & - & - \\
IRS 16 C & $0.57 \pm 0.12$ & $0.0027 \pm 0.0008$ & $-7.57 \pm 1.19$ & $94 \pm 10$ \\
IRS 16 NE & $1.15 \pm 0.16$ & $0.0084 \pm 0.0024$ & $-7.62 \pm 1.31$ & $147 \pm 21$ \\
IRS 16 SW & $0.38 \pm 0.09$ & $0.0026 \pm 0.0008$ & $-4.30 \pm 1.18$ & $137 \pm 10$ \\
\hline
\end{tabular}
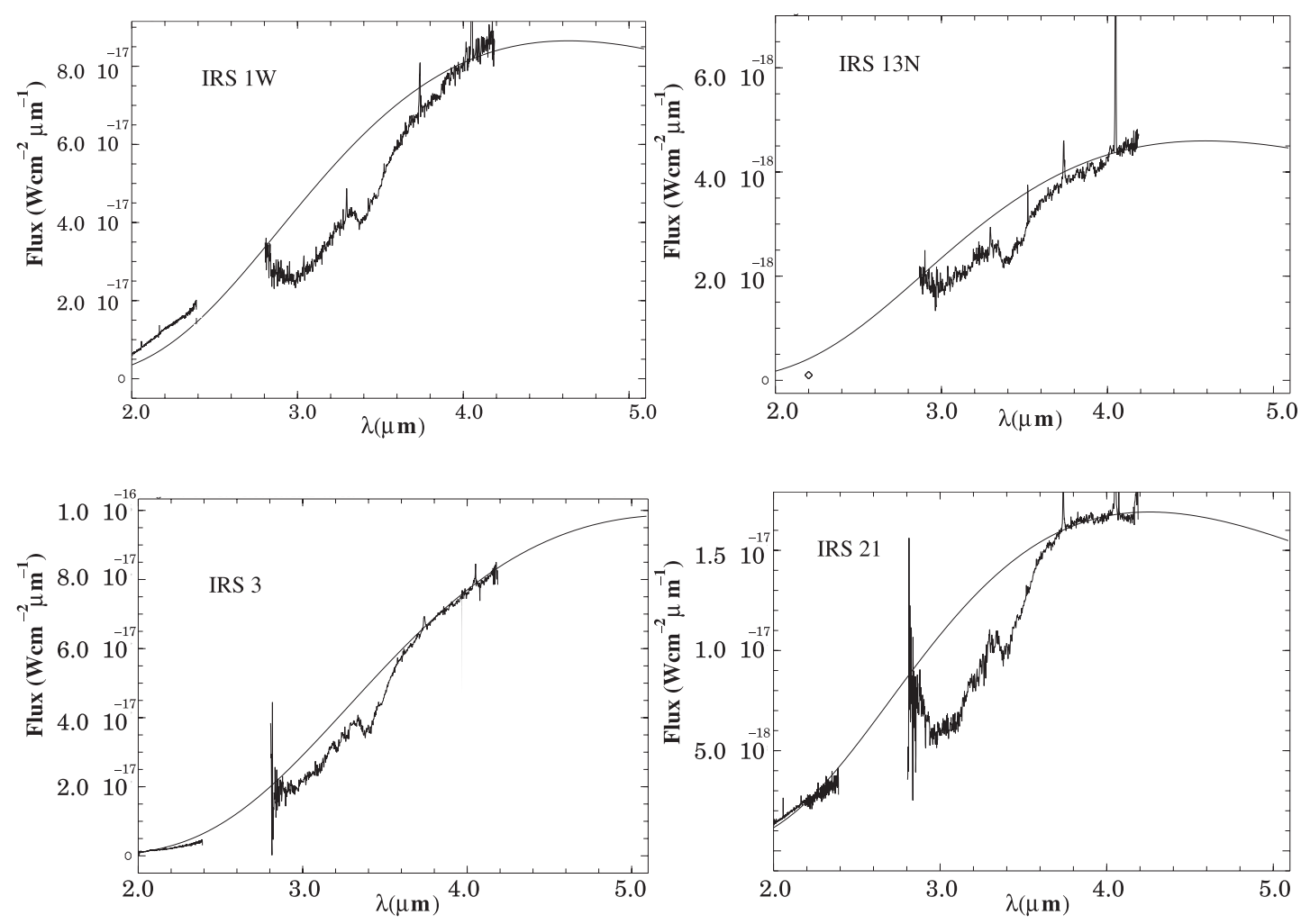

Fig. 2. Best fits of the $K$ - and $L$-band spectra with single reddened blackbody continua. When no $K$-band spectrum was available, the flux density at $2.2 \mu \mathrm{m}$ represented by a diamond was considered in the fitting procedure. 
J. Moultaka et al.: Interstellar matter and circumstellar matter in the central parsec of the Milky Way, Online Material p 4
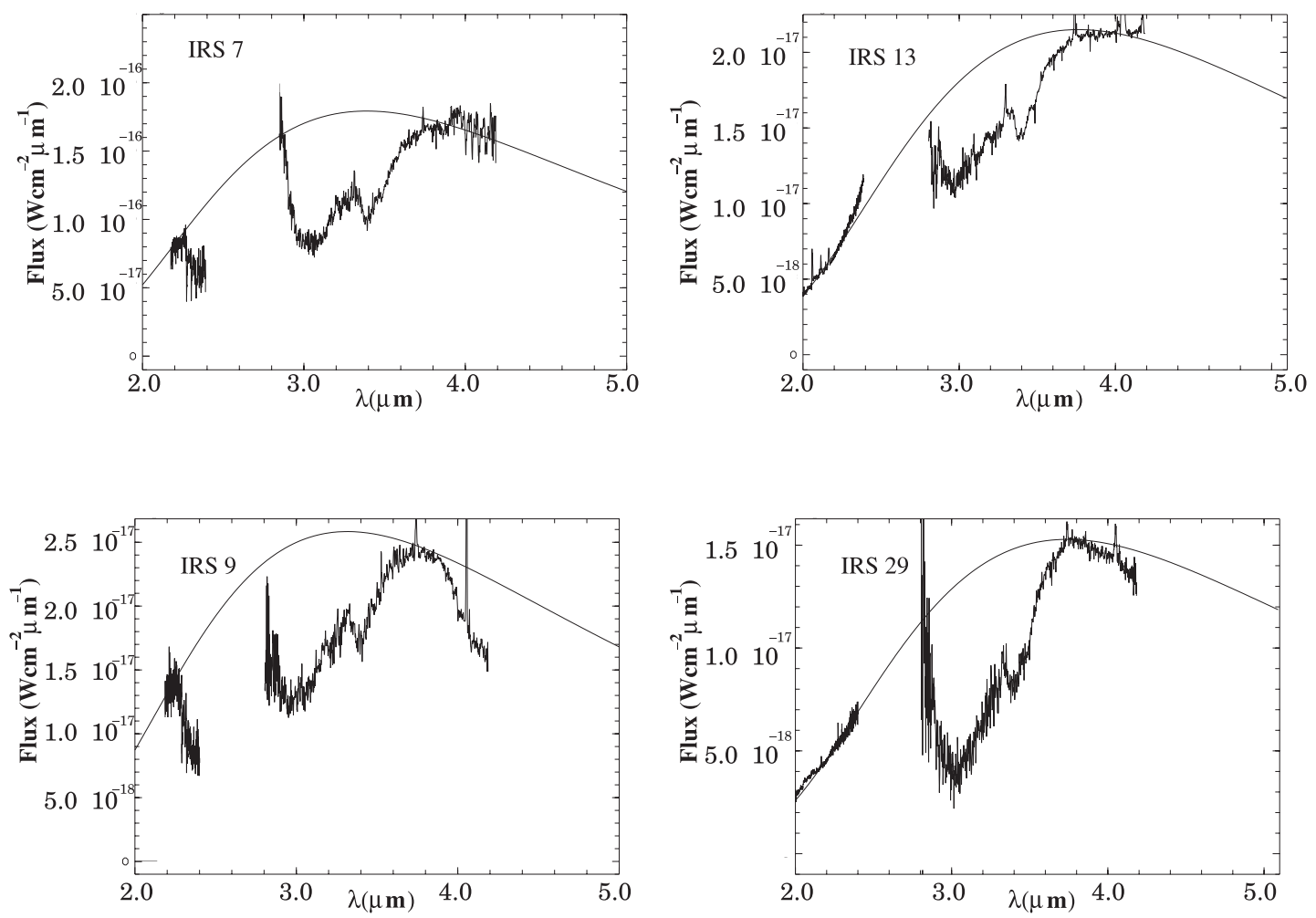

Fig. 3. Fitting spectra with single reddened blackbody continua (cont.).
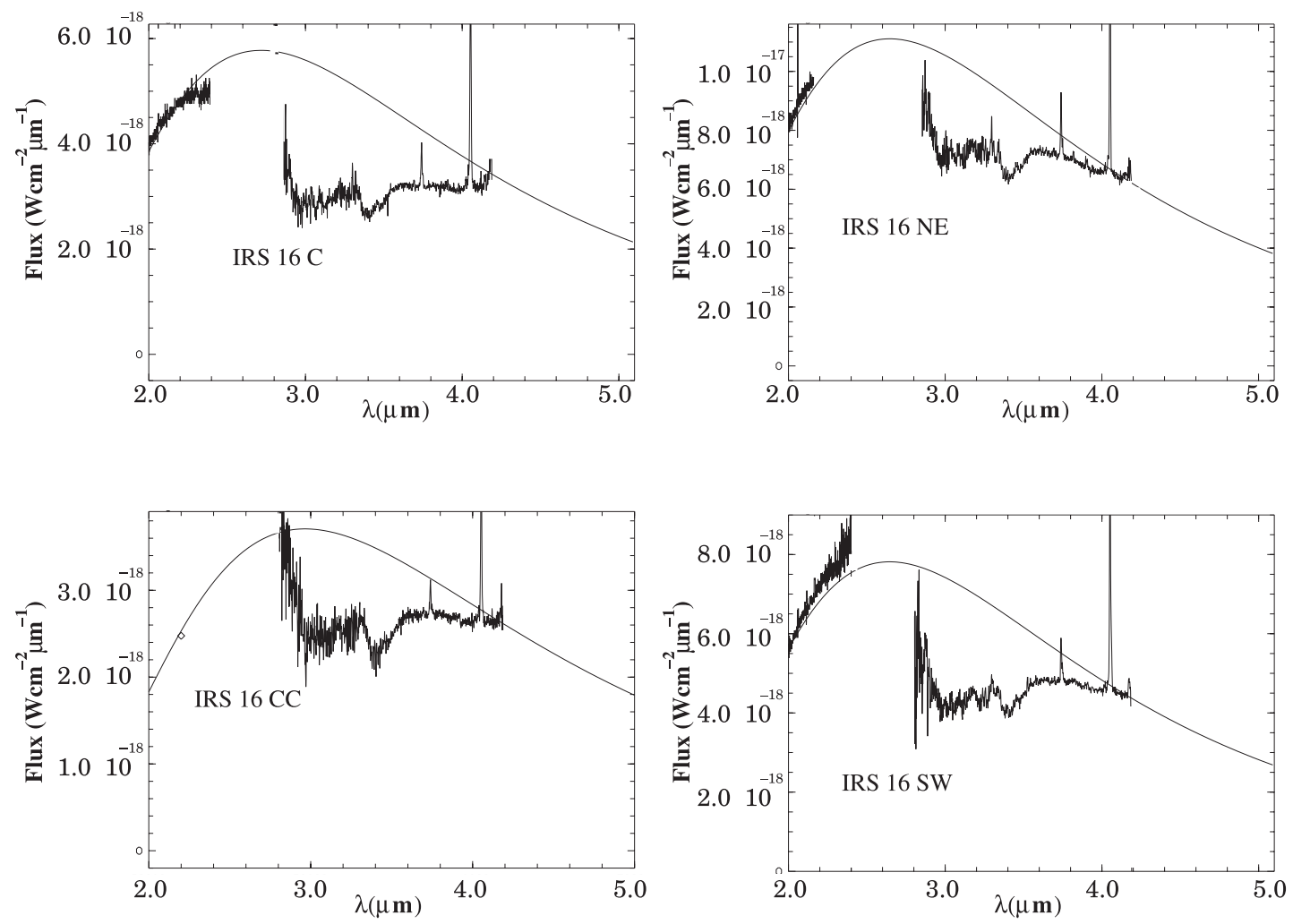

Fig. 4. Fitting spectra with single reddened blackbody continua (cont.). 
J. Moultaka et al.: Interstellar matter and circumstellar matter in the central parsec of the Milky Way, Online Material p 5

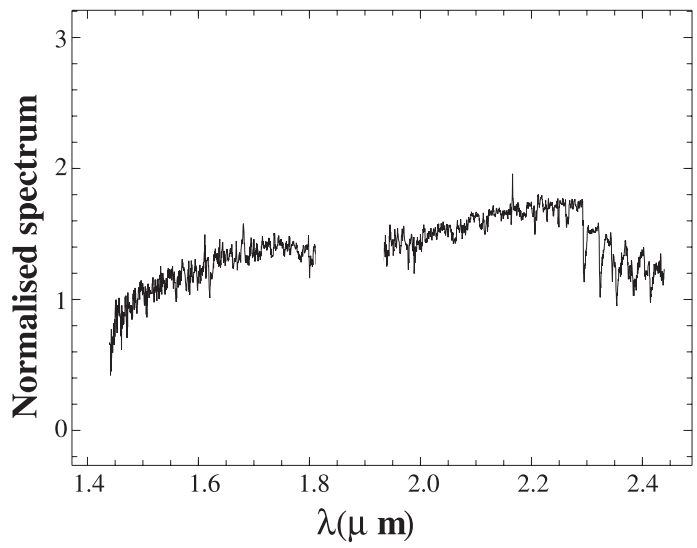

Fig. 6. The $H$ - and $K$-band spectrum of the late-type CO-star whose $L$-band spectrum is used for the line of sight absorption correction.

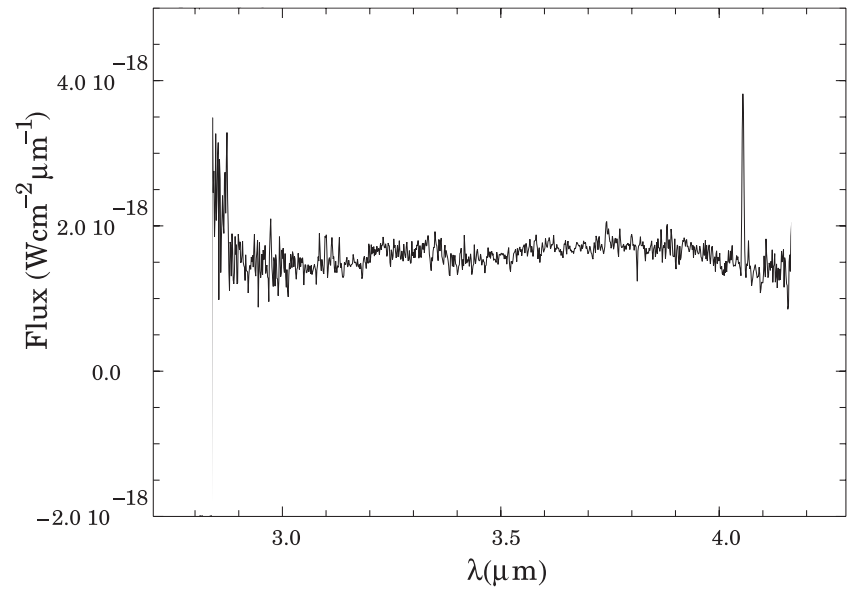

Fig. 7. The $L$-band spectrum of the late-type CO-star used for the line of sight absorption correction. 
J. Moultaka et al.: Interstellar matter and circumstellar matter in the central parsec of the Milky Way, Online Material p 6
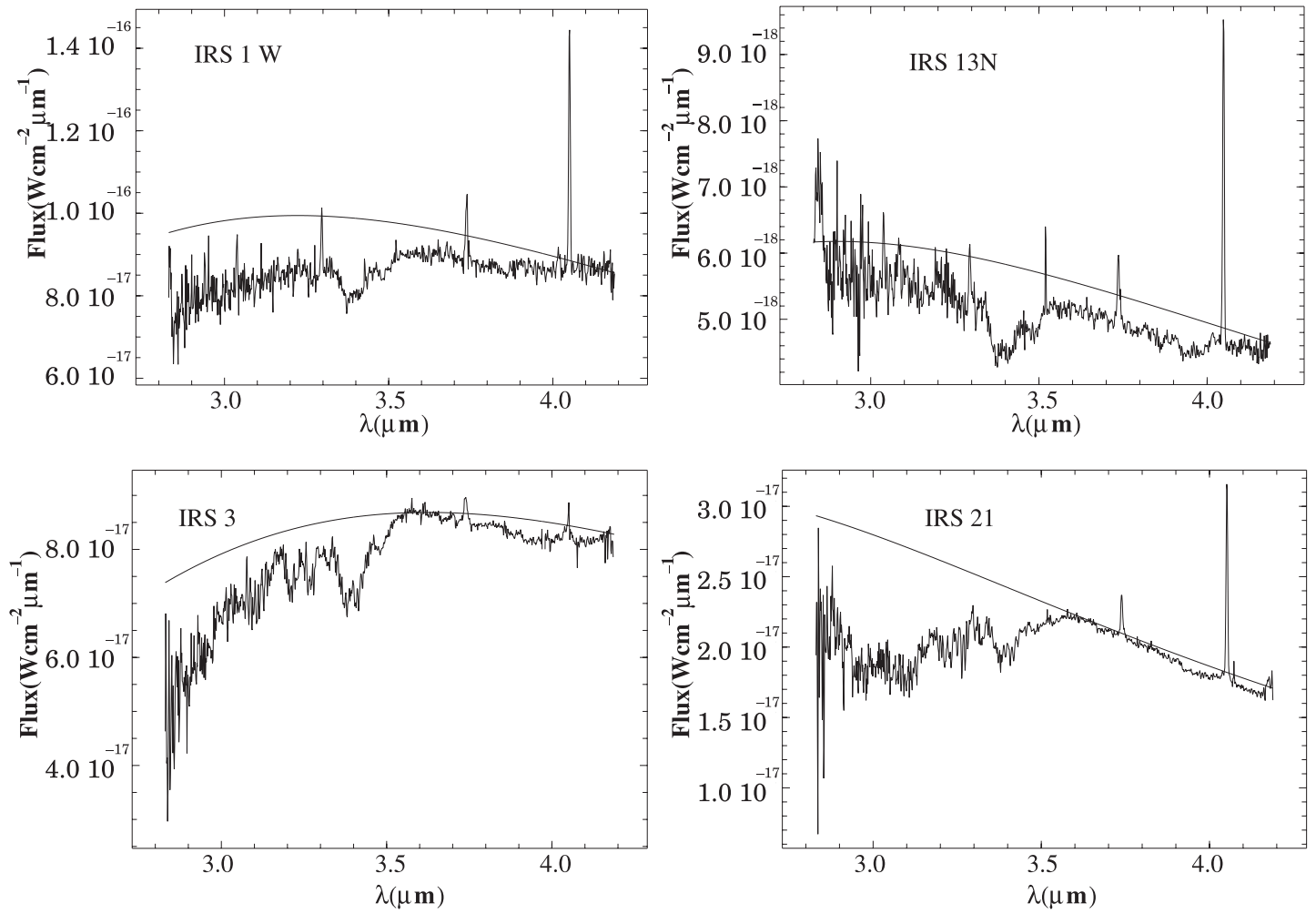

Fig. 9. $L$-band spectra corrected for line of sight absorption using the optical depth spectrum shown in Fig. 8. Blackbody continua (not reddened) of temperatures listed in Table 1 are also plotted for comparison.
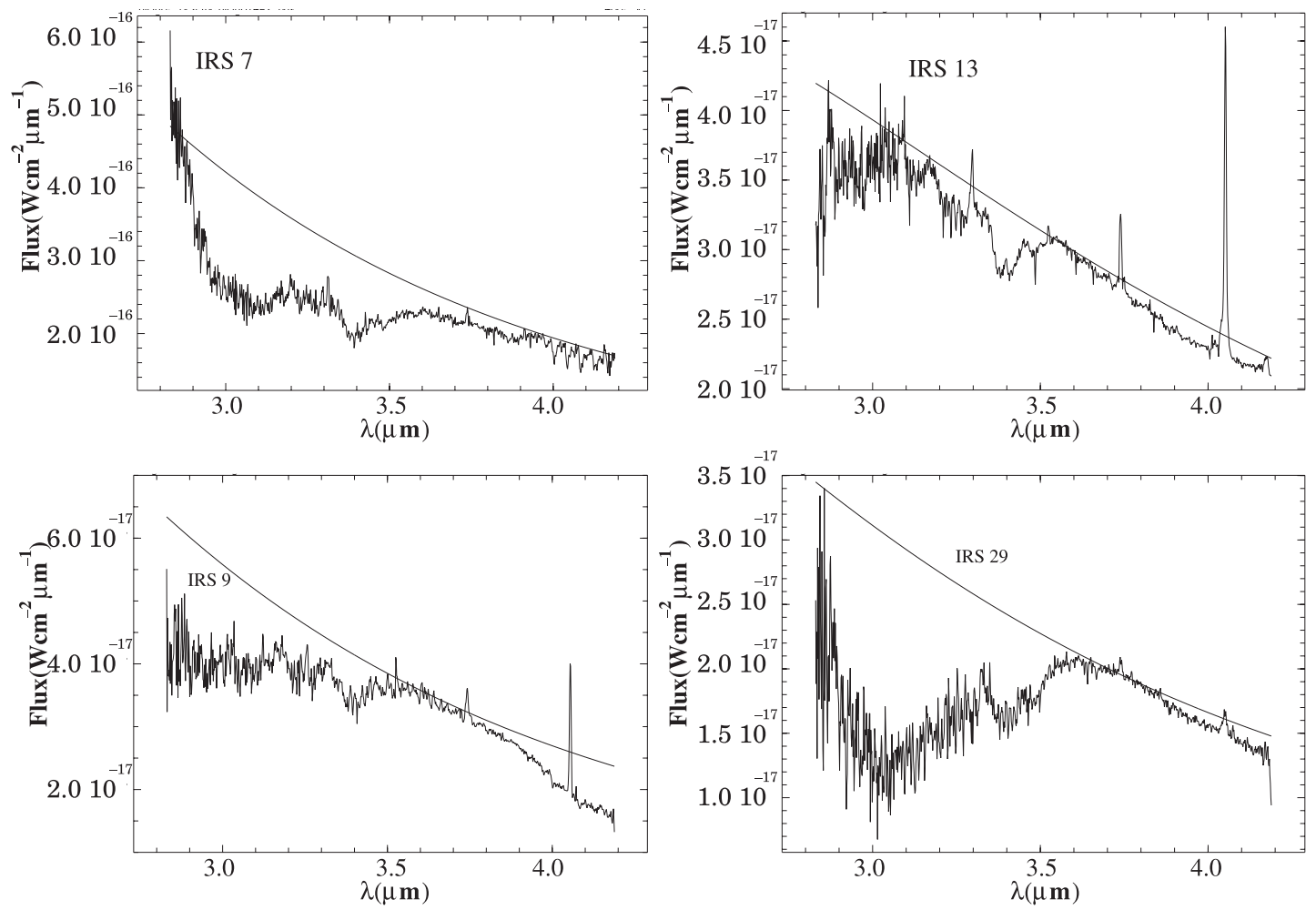

Fig. 10. $L$-band spectra corrected for line of sight absorption (cont.). 
J. Moultaka et al.: Interstellar matter and circumstellar matter in the central parsec of the Milky Way, Online Material p 7
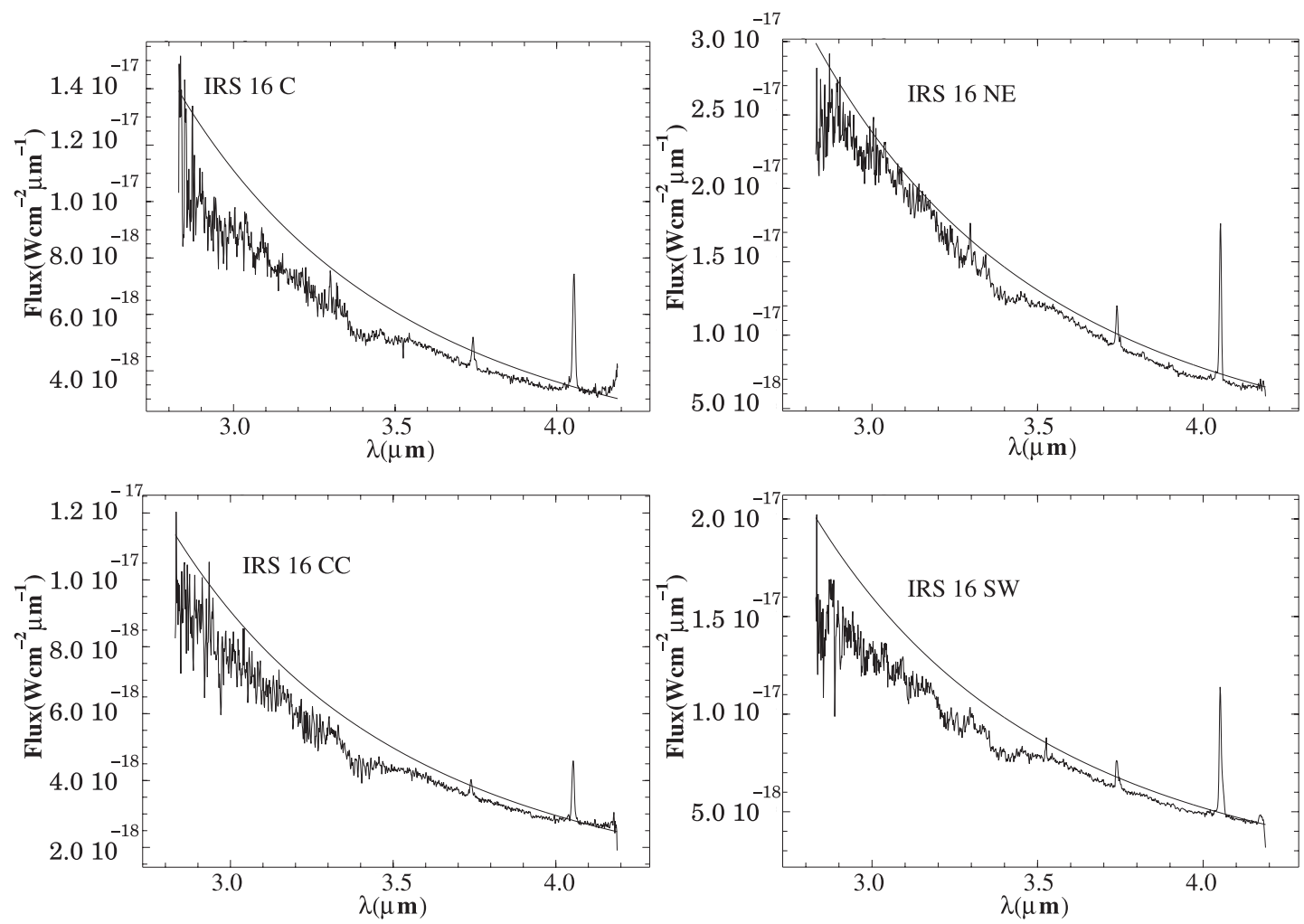

Fig. 11. $L$-band spectra corrected for line of sight absorption (cont.). 